Comparative Population Studies

Selected Articles in German Vol. 41 (2016): 101-136

(Erstveröffentlichung: 22.08.2017)

\title{
Einfluss regionaler Faktoren auf die Kohortenfertilität: Neue Schätzwerte auf Kreisebene in Deutschland ${ }^{*}$
}

\author{
Martin Bujard, Melanie Scheller
}

Zusammenfassung: Betrachtungen des regionalen Kontexts haben eine lange Tradition in der Fertilitätsforschung. Allerdings liegen für Deutschland kreisspezifische Fertilitätsdaten bisher lediglich für die zusammengefasste Geburtenziffer (TFR) vor, nicht jedoch zur endgültigen Kinderzahl (Kohortenfertilität, CTFR). Auf Basis von Zensus 2011 und Geburtenstatistik werden Schätzwerte für die CTFR auf Kreisebene generiert und Einflussfaktoren ihrer Variation analysiert.

Zunächst wird die CTFR der Geburtskohorten 1969-72 für alle 402 Kreise berechnet. Die geschätzte CTFR variiert erheblich zwischen den Kreisen bei einer Spannbreite von 1,05 bis 2,01. Sie unterscheidet sich deutlich von den bekannten Werten der TFR. Diese Unterschiede beruhen hauptsächlich auf Verzerrungen durch die Timing-Komponente der TFR, die in Ostdeutschland, bei Universitätsstädten und im Stadt-Land-Vergleich auffällig sind. Daher ermöglichen die geschätzten CTFRWerte einen weitaus besseren Einblick in regionale Fertilitätsunterschiede.

Im zweiten Schritt werden die Unterschiede der neu gewonnenen CTFR auf Kreisebene mittels linearer Regressionen analysiert. Die drei Kompositionseffekte geringe Akademikerinnenquote, hoher Katholiken- und hoher Migrantenanteil tragen signifikant zu einer höheren CTFR in westdeutschen Kreisen bei. Allerdings spielen regionale Opportunitäten ebenfalls eine wichtige Rolle: Eine geringe Bevölkerungsdichte, das Angebot relativ großer Wohnungen und ein Männerüberschuss sind deutlich mit höherer Fertilität assoziiert, ebenso ökonomische Faktoren wie traditionelle Wirtschaftsstruktur und niedrige Arbeitslosigkeit. Insgesamt zeigen die Analysen, dass regionale Faktoren einen hohen Einfluss auf die Fertilität haben.

Schlagwörter: Kohortenfertilität · Bevölkerungsgeographie · Regionaler Kontext · Urbanisierung $\cdot$ Deutschland $\cdot$ Zensus

\footnotetext{
Dieser Artikel enthält ergänzende Materialien in einem Online-Anhang: DOI: 10.12765/CPoS 2017-08de,

URL: http://www.comparativepopulationstudies.de/index.php/CPoS/article/view/278/251.
} 


\section{$1 \quad$ Einleitung}

Ein großer Teil der Fertilitätsforschung lässt sich in zwei Gruppen aufteilen: Solche, die Individualdaten untersuchen und solche, die auf Makroebene Länder vergleichen. Bei beiden Ansätzen werden regionale Faktoren oftmals ausgeklammert, was angesichts der erheblichen regionalen Unterschiede im generativen Verhalten (Basten et al. 2011) zu Verzerrungen aufgrund von Selection-Bias oder Whole-NationBias (Rokkan 1970) führt. Da bspw. innerhalb Deutschlands die Varianz der Fertilität auf Kreisebene sehr hoch ist und sich auch die lokalen Opportunitätsstrukturen hinsichtlich ökonomischer, politischer und kultureller Faktoren stark unterscheiden (BBSR 2015), ist es essenziell, die Fertilitätsforschung um kreisspezifische Faktoren zu erweitern.

Die Ursachen regional divergierender Fertilitätsraten können dabei sehr unterschiedlich sein: Die regionale Varianz lässt sich zum Teil auf Kompositionseffekte (Hank 2002; Hank/Huinink 2015) und zum Teil auf unterschiedliche regionale Opportunitätsstrukturen (Überblick: Basten et al. 2011) oder historisch gewachsene kulturelle Prägungen (K/üsener/Goldstein 2014) zurückführen. Der in der Literatur am stärksten belegte regionale Faktor ist zweifellos der Urbanisierungsgrad (Kulu et al. 2007; Kulu/Boyle 2009; Trovato/Grindstaff 1980), hinter dem sich jedoch unterschiedliche Mechanismen verbergen können (Sharlin 1986); insbesondere Faktoren wie Wohnraum (Fiori et al. 2014; Kulu 2013) und selektive Binnenmigration (Courgeau 1989; Kulu/Milewski 2007). Auch regionale Unterschiede bezüglich Familienpolitik (Baizan 2009; Rindfuss et al. 2007), ein hoher Grad an Religiosität (Sobotkal Adigüze/ 2002) und niedrige Arbeitslosigkeitsraten (Kravdal 2002) sind mit höheren Fertilitätsraten assoziiert. Allerdings ist die Befundlage in Europa teilweise widersprüchlich, was zum einen an länderspezifischen Unterschieden liegt und zum anderen an den spezifischen Forschungsdesigns und Datensätzen. Viele regionale Analysen decken nur einen Teil der potenziellen Faktoren ab, indem wenige regionale Variablen in ereignisanalytischen Mehrebenenmodellen getestet werden. Dieser State of the Art der Forschung ermöglicht zwar eine kausale Interpretation, jedoch oft zum Preis eines Selection-Bias, wonach die Vielfalt potenzieller regionaler Variablen und ihre Interaktionen ausgeblendet werden.

Um Fertilitätsunterschiede auf regionaler Ebene - und insbesondere auf Kreisebene - für Deutschland quantifizieren und ihre Faktoren analysieren zu können, war die Datenlage bisher begrenzt, denn es lagen keine Daten zur Kohortenfertilität auf Kreisebene vor. Es gibt zwar detaillierte Daten zur zusammengefassten Geburtenziffer (TFR, auch bekannt als PTFR) auf Kreisebene (Grünheid 2015; BBSR 2015), allerdings ist die TFR als abhängige Variable weniger geeignet, da sie durch die Timing-Komponente (Bongaarts/Feeney 1998; Schoen 2004) verzerrt ist. Die Kohortenfertilitätsraten (Cohort Total Fertility Rate, CTFR) lassen sich bezogen auf regionale Unterschiede besser analysieren. Die CTFR (bzw. die durchschnittliche Kinderzahl pro Frau) auf Kreisebene und die damit verbundenen regionalen Analysen stellen eine erhebliche Forschungslücke für demografische und geografische Analysen dar. Der Zensus 2011 hat zwar nicht die biologische Kinderzahl erfragt, 
bietet jedoch das Potenzial, mittels demografischer Schätzmodelle diese Forschungslücke zu schließen.

Dieser Artikel hat zwei Ziele:

- Einen Schätzwert zur CTFR für alle 402 deutschen Kreise zu berechnen auf Basis der aus dem Zensus 2011 ermittelten Kinderzahl pro Frau im Haushalt sowie

- einen breiten Überblick zu regionalen Erklärungsfaktoren zu generieren und deren Interaktionen im Kontext sozialstruktureller Faktoren zu analysieren.

Für die Berechnungen der CTFR sind hohe Fallzahlen erforderlich, da auch in kleineren Kreisen ausreichend Fälle für jahrgangsspezifische Kinderzahlen vorhanden sein müssen. Dies ist selbst beim Mikrozensus (MZ) mit 688.931 Personen im Berichtsjahr 2012 nicht ausreichend gegeben. Der Zensus ermöglicht hier Neuland zu betreten: Es wurden 7,9 Mio. Personen, also rund $10 \%$ der deutschen Bevölkerung, befragt. Da der Zensus Daten zur Kinderzahl nicht direkt erfasst, wird als Annäherung die Kinderzahl im Haushalt (Familienebene) verwendet, um einen Schätzwert der CTFR für alle 402 deutschen Kreise zu berechnen. Durch eine Identifizierung der Frauenjahrgänge, die einerseits die reproduktive Lebensphase weitgehend abgeschlossen haben und bei denen andererseits die Kinder überwiegend noch im Elternhaus leben, lässt sich ein möglichst genauer Schätzwert für die CTFR auf Kreisebene generieren. Um die jahrgangsspezifische Unterschätzung im Vergleich zur amtlichen Bevölkerungsstatistik zu berechnen, sind Extrapolationen auf Basis der amtlichen Bevölkerungsstatistik sowie ein nach Ost- und Westdeutschland differenzierter Multiplikator notwendig. ${ }^{1}$ Die so errechneten CTFR-Werte auf Kreisebene sind in Form von Grafiken und Tabellen im Anhang sowie in einem Excel-Dokument im Online-Anhang dargestellt.

Die Faktoren der regionalen Fertilitätsunterschiede werden durch lineare Regressionsanalysen auf der Makroebene operationalisiert, wobei die 402 Kreise die Fälle und die zuvor berechnete CTFR die abhängige Variable darstellen. Um Kompositionseffekte abbilden zu können, werden soziodemografische Merkmale wie Angaben zu Bildung, Religion, Migrationshintergrund und Lebensform basierend auf eigenen Auswertungen mit den Mikrodaten des Zensus aggregiert. Aus der Datenbank INKAR ${ }^{2}$ (BBSR 2015) werden kreisspezifische Daten zu Arbeitsmarkt, Wirtschaftsstruktur, Frauenerwerbstätigkeit, Wohlstand, Urbanisierung, Familienpolitik, Wohnraum und Geschlechterproportionen für die Analyse aufbereitet. Dies ermöglicht eine gemeinsame Betrachtung regionaler und soziodemografischer Einflussfaktoren der Fertilität.

1 Zu Ostdeutschland zählen in dieser Berechnung die Neuen Bundesländer sowie Berlin.

2 INKAR ist das Akronym für "Indicators, Maps and Graphics on Spatial and Urban Monitoring". 


\section{Forschungsstand}

\subsection{Fertilitätsdaten der Kreise in Deutschland}

Die TFR wird von den Statistischen Landesämtern auf Kreisebene berechnet, wobei die altersspezifischen Fertilitätsraten (ASFR) für jedes Alter in einem bestimmten Jahr addiert werden. Für einzelne Jahre wie 2013 (Grünheid 2015; ohne Thüringen) liegen diese Werte für Gesamtdeutschland vor. Datenreihen zur TFR werden von INKAR (BBSR 2015) berechnet, die auf Daten der Statistik der Geburten und Sterbefälle des Bundes und der Länder und der Regio-Datenbank von Eurostat beruhen. Der von INKAR verwendete Algorithmus berechnet die TFR jedoch nicht auf Basis der ASFR sondern auf Basis von Fünfjahresaltersgruppen, wie 15-19 Jahre, 20-24 Jahre usw. (Wir nutzen diese Daten für einen Vergleich mit der berechneten CTFR in Abschnitt 4.1).

Daten zur Kohortenfertilität auf Kreisebene in Deutschland existieren bisher nicht. Die amtliche Geburtenstatistik berechnet die CTFR auf Basis von ASFR für jedes einzelne Jahr und jede Kohorte, wobei sich das Alter aus der Differenz zwischen Geburtsjahr und Berichtsjahr errechnet (Statistisches Bundesamt 2014). Jedoch bietet sie nur CTFR für Gesamtdeutschland bzw. nach Ost- und Westdeutschland differenziert. Sozialwissenschaftliche Erhebungen und der MZ 2012, die die biologische Kinderzahl erheben, haben zu geringe Fallzahlen für die Berechnung der kreisspezifischen Kohortenfertilität. Der MZ 2012 hat immerhin 688.931 Fälle, die Frauenjahrgänge 1969-1972 haben jeweils im Schnitt 4.569 Fälle, was pro Kreis durchschnittlich 13 Fällen entspricht. Der Zensus hat 7,9 Mio. Fälle, dabei durchschnittlich 48.484 Fälle für einen der Frauenjahrgänge 1969-1972, was pro Kreis im Schnitt 121 Fällen entspricht. Nimmt man die vier Jahrgänge zusammen, beträgt die durchschnittliche Fallzahl immerhin 482 pro Kreis.

Die CTFR auf Kreisebene ist ein wertvoller Indikator, um regionale Fertilitätsunterschiede und die sie beeinflussenden regionalen Opportunitäten zu messen, da sie die Fertilität innerhalb der gesamten reproduktiven Lebenszeit berücksichtigt. Dagegen handelt es sich bei der TFR um ein andersartiges Konzept, bei dem neben dem Quantum auch der Zeitpunkt der Geburten einfließt. Auf Kreisebene ist die TFR durch das Ausmaß der Binnenmigration junger Frauen, insbesondere von Studentinnen sowie die unterschiedlich starken Geburtenaufschübe so verzerrt, dass die Interpretierbarkeit begrenzt ist. Der Timingeffekt ist problematisch (Schoen 2004), da sich der Aufschub der Geburten bzw. das durchschnittliche Alter bei der Geburt regional erheblich unterscheiden. Universitätsstädte mit einem hohen Akademikeranteil wie Heidelberg, Münster oder Würzburg haben ein durchschnittliches Geburtsalter (MAC) von 32,5-33,3 Jahren. Im Vergleich dazu liegt das MAC im Jahr 2013 in ländlichen Regionen wie Mecklenburgische Seenplatte und VorpommernRügen bei 27,0-27,4 Jahren (Grünheid 2015). 


\subsection{Forschungsstand zu regionalen Fertilitätsunterschieden}

Bei den regionalen Fertilitätsunterschieden lassen sich zwei grundsätzlich verschiedene Erklärungen anführen: Sie können auf Kompositionseffekten oder auf regionalen Opportunitätsstrukturen beruhen (Basten et al. 2011; Boyle et al. 2007; Firoi et al. 2014; Hank 2002; Hank/Huinink 2015; Kulu/Boyle 2009; Kulu et al. 2007; Trovato/ Grindstaff 1980). Hinsichtlich dieser Grundsatzfrage stimmen zwar die meisten Autoren der These zu, dass beide Erklärungen sich ergänzen. Allerdings finden einige Autoren eher Kompositionseffekte relevant (Hank 2002; ${ }^{3}$ Hank/Huinink 2015), während andere regionale Kontextfaktoren betonen (Kulu et al. 2007; Kulu/Boyle 2009). Diese Unterscheidung ist für die Bedeutung regionaler Analysen wichtig, denn sowohl der Whole-Nation-Bias bei Makroanalysen (Rokkan 1970) als auch der Selection-Bias durch die Ausblendung regionaler Faktoren bei Individualanalysen sind umso größer, je stärker regionale Kontextfaktoren wirken.

Kompositionseffekte führen v.a. dann zu regional unterschiedlichen Fertilitätsraten, wenn in bestimmten Regionen Personen mit solchen sozialstrukturellen Merkmalen überrepräsentiert sind, die ein spezifisches Fertilitätsverhalten aufweisen. In Ländern, bei denen höhere Bildungsabschlüsse mit niedrigerer Fertilität assoziiert sind, ist die Fertilität in Regionen deutlich niedriger, in denen besonders viele Akademiker und wenige Frauen mit niedrigem Bildungsabschluss leben (Hank/Huinink 2015). Ähnliches gilt für den sozioökonomischen Status (Kulu 2013). Kompositionseffekte können auch auf Migrantengruppen bzw. ethnischen Minoritäten beruhen, die in bestimmten Regionen überrepräsentiert sind und ein abweichendes Fertilitätsmuster aufweisen. Die Fertilität von Migranten unterschiedlicher Herkunftsländer ist sehr heterogen. In Deutschland und Großbritannien, wo die Mehrzahl der Migranten aus Ländern mit deutlich höherer Fertilität kommt, wie Türkei oder Pakistan, ist der Effekt von Migranten positiv (Hank/Huinink 2015; Kulu/Washbrook 2014). Andere Studien zeigen keinen Einfluss von internationalen Migranten, v.a. im Vergleich zu den deutlichen Effekten der Binnenmigration (Michielin 2002). Individuelle Merkmale bezüglich der Lebensform (Hank 2003) oder der Erwerbstätigkeit erweisen sich auch in vielen Analysen als signifikant (Hank/Huinink 2015). Ihre Interpretation als Kompositionseffekte ist jedoch schwierig, da diese Faktoren auch Folge von Fertilitätsereignissen oder zumindest unterschiedlich stark ausgeprägten Kinderwünschen sein können.

Regionale Kontextfaktoren beruhen auf lokalen Opportunitätsstrukturen (ausführlich: Basten et al. 2011), die sich in Bezug auf Urbanisierung, Wohnraum, Kultur, Familienpolitik und Arbeitsmarkt unterscheiden.

Ein zentraler Faktor ist der Urbanisierungsgrad (Courgeau 1989; Kulu/Boyle 2009; Kulu et al. 2007; Kulu 2013; Trovato/Grindstaff 1980), der bereits in der historischen Demografie und der ersten demografischen Transition negativ mit Fertilität verbunden war (Andorka 1978; Sharlin 1986; Wolf 1912). Die Bevölkerungsdichte weist in

3 Allerdings weist Hank auf weiteren Forschungsbedarf, u.a. durch eine Nutzung groß-skalierter Individualdatensätze, hin (Hank 2002: 296). 
einem internationalen Vergleich von 145 Ländern einen negativen Zusammenhang mit Fertilität auf (Lutz et al. 2006), wobei dies nur bedingt auf Europa zutrifft. Die Interpretation des Einflusses des Urbanisierungsgrades für Fertilitätsentscheidungen ist jedoch kompliziert, da die dahinter stehenden Mechanismen vielfältig sind. Sharlin (1986: 236) bezeichnet Stadt-Land-Unterschiede als "code phrase for an array of social, economic, and cultural differences and changes". Die Mechanismen hinter dem Urbanisierungseffekt lassen sich in lokale Opportunitätsstrukturen, selektive Binnenmigration, kulturelle Faktoren (inklusive dem Timing von Wandlungsprozessen) und Kompositionseffekte differenzieren:

- Bestimmte lokale Opportunitätsstrukturen unterscheiden sich typischerweise zwischen Städten und ländlichen Regionen: In Städten ist der Wohnraum knapper und teurer sowie der Arbeitsmarkt von hoher Spezialisierung und Wissensbasierung geprägt; beide Faktoren sind mit niedriger Fertilität assoziiert. In ländlichen Kreisen dagegen können Grünflächen zur Naherholung und weniger Autoverkehr eher zu einer kinderfreundlichen Wohnumgebung beitragen. Es lohnt sich jedoch aus zwei Gründen die Faktoren einzeln zu anaIysieren: Erstens, um die tatsächlichen Wirkungsfaktoren zu erhalten, die sich hinter der Urbanisierung verbergen. Zweitens ist eine Differenzierung der lokalen Opportunitätsstrukturen in Städten sinnvoll, da es auch urbane Räume mit familienfreundlichen Wohnumgebungen gibt. Mehrere Studien zeigen, dass die Fertilität in Vororten höher ist als im Stadtzentrum (Boyle et al. 2007; Kulu/Boyle 2009; Kulu/Washbrook 2014).

- Selektive Binnenmigration (Courgeau 1989; Huinink/Wagner 1989; Kulu/Milewski 2007; Michielin 2002) verstärkt Stadt-Land-Unterschiede in der Fertilität. Häufig ziehen Paare kurz vor oder nach der Familiengründung von Städten aufs Land und Kinderlose eher in Städte. Courgeau (1989) zeigt für Frankreich, dass die Binnenmigration von Stadt nach Land mit höherer Fertilität einhergeht (und vice versa). Dies zeigt er nicht nur für die Familiengründung, sondern auch beim Übergang zu höheren Paritäten. Es wird argumentiert, dass Frauen ihr Verhalten an die neue Umgebung anpassen bzw. eine Umgebung entsprechend ihrer Kinderwünsche auswählen, wobei der Wohnungsmarkt eine wichtige Rolle spielt. Da manche Vororte zur Stadt und andere zu angrenzenden ländlichen Kreisen gezählt werden, sind Abgrenzungsprobleme hier virulent.

- Kulturelle Unterschiede zwischen städtischen und ländlichen Kreisen sind auf unterschiedliche sozio-kulturelle Milieus zurückzuführen. In urbanen Milieus ist ein städtischer Lebensstil verbreitet, der von vielen Lebensoptionen außerhalb von Familie geprägt ist. Dabei interagieren urbaner Lebensstil und lokale Opportunitätsstrukturen miteinander. Der Einfluss dieser Milieus und des sozialen Lernens ist bei der Familiengründung größer als bei dem Übergang zu weiteren Paritäten (Fiori et al. 2014). Stadt-Land-Unterschiede können auch die Avantgarde und Nachzügler in historischen Wandlungsprozessen, wie dem ersten demografischen Übergang, abbilden (Coale/Watkins 1986).

Selektive Binnenmigration findet jedoch nicht nur im Stadt-Land-Bezug statt, sondern generell zwischen unterschiedlichen Kreisen mit unterschiedlichen regionalen Kontexten. Kulu und Milewski (2007) haben eine elaborierte Systematik vorgelegt, bei der sie vier Hypothesen für das Fertilitätsverhalten bei Migration differenzieren: Der Sozialisationshypothese zufolge bleibt die Fertilität nach einem 
Umzug von erwachsenen Personen konstant, da die Präferenzen bereits vor dem Umzug gebildet worden sind. Dagegen postuliert die Adaptionshypothese, dass der regionale Kontext ausschlaggebender ist und sich die Fertilität an das Muster ihrer neuen Umgebung anpasst. Die Selektionshypothese geht davon aus, dass ein Umzug als Folge von spezifischen Fertilitätspräferenzen vorgenommen wird (für Großbritannien: Kulu/Washbrook 2014). Die Disruptionshypothese besagt, dass direkt nach einem Umzug die Fertilität niedriger ist; diese Hypothese ist weniger für Binnenmigration als für internationale Migration (abgesehen vom Familiennachzug) plausibel. Anders als die Befunde von Courgeau (1989) für Frankreich, Huinink und Wagner (1989) für Deutschland und Michielin (2002) für Italien zeigen neuere AnaIysen für den Übergang zum zweiten und dritten Kind für Großbritannien (Fiori et al. 2014) und für die ersten drei Paritäten in Finnland (Kulu 2013) keinen Effekt der selektiven Migration.

Der Wohnraum erweist sich in mehreren Studien als signifikanter regionaler Kontextfaktor (Fiori et al. 2014; Kulu/Vikat 2007; Kulu/Boyle 2009; Kulu 2013). Kulu zeigt für Finnland, dass das Wohnen in freistehenden Häusern und Reihenhäusern beim Übergang zum ersten Kind einen deutlichen Effekt hat, der sogar den häufigeren Übergang in ländlichen Gebieten erklärt (Kulu 2013: 904). Für Finnland konnte auch ein wahrscheinlicherer Übergang zum dritten Kind bei Einfamilienhäusern gezeigt werden (Kulu/Vikat 2007). Für Großbritannien finden Fiori et al. (2014) einen positiven Effekt von Wohnungen mit mehr als fünf Räumen lediglich beim Übergang zum zweiten Kind.

Kulturelle Unterschiede auf regionaler Ebene, die historisch gewachsen und oft bereits vor dem gegenwärtigen politischen System entstanden sind (vgl. Klüsener) Goldstein 2014), können sich auf regionale Fertilitätsunterschiede auswirken. Die Bedeutung der Kultur in Relation zu sozioökonomischen Unterschieden betonen die Autoren des „European Fertility Project" (Coale/Watkins 1986). Während ein gewisser Grad an Modernisierung eine Voraussetzung für den prätransitionellen Geburtenrückgang war, wurde der Zeitpunkt primär vom regionalen kulturellen Setting beeinflusst (Knodel/van de Walle 1986). Dies gilt nicht für die konfessionelle Prägung in großen Städten Preußens; zudem spielen Ansteckungseffekte zwischen geografischen Nachbarregionen eine Rolle (Goldstein/K/üsener 2014). Auch neuere Studien weisen auf den Einfluss von regional unterschiedlichen sozialen Milieus auf Fertilität hin (Fulda 2015). Andererseits ist die Kultur im Sinne von fertilitätsbezogenen Einstellungen zu einem gewissen Teil national, durch Sprache und Massenmedien, geprägt (Lesthaeghe 1995).

Die Konfessionen und die unterschiedliche Religiosität, beispielsweise durch wöchentliche Kirchbesuche messbar, sind ein Ankerpunkt für kulturelle Analysen. Mehrere Studien zeigen, dass eine hohe Kirchgangsquote zur Erklärung regionaler Fertilitätsunterschiede beiträgt (Kemper 1991; Sobotka/Adigüze/ 2002). Während vor einem Jahrhundert die höhere Fertilität bzw. geringe Neigung zur Verhütung von Katholiken betont wurde (Wolf 1912; vgl. Goldstein/K/üsener 2014), wird Katholizismus in neueren Publikationen mit niedriger Fertilität infolge von familienpolitischen Defiziten im internationalen Vergleich assoziiert (Castles 2003). Analysen von 28 OECD-Ländern zeigen, dass die Protestantenquote zwischen 1987 und 2006 
positiv mit der TFR assoziiert ist, wobei Kinderbetreuungs- und Protestantenquote in multivariaten Regressionen eine ähnliche Varianz erklären (Bujard 2011). Dabei ist in vielen Ländern nur eine Konfession dominierend. Deutschland ist hier ein interessanter Fall, da es Kreise gibt, die primär katholisch (u.a. in Südbayern) und solche, die primär protestantisch (u.a. in Schleswig-Holstein) sind. Dabei unterscheiden sich die Anteile auch innerhalb der einzelnen Bundesländer teilweise deutlich. Um den kirchlichen Einfluss zu messen, ist neben der Konfession auch die Religiosität maßgeblich, wobei Ostdeutschland - nicht nur aufgrund des DDR-Regimes in besonderem Maße säkular ist (K/üsener/Goldstein 2014). Studien, die regionale Fertilitätsunterschiede in Deutschland im Kontext von Kirchen und Religiosität untersuchen, sind selten. Kemper (1991) zeigt für 31 westdeutsche Regierungsbezirke anhand eines Pfadmodells einen direkten positiven Einfluss der Kirchenbesuchsrate und einen indirekten negativen der Protestantenquote.

Einige neuere Studien zum Einfluss der Familienpolitik auf die Fertilität auf Länderebene zeigen einen positiven Effekt (Bujard 2011; Luci-Greulich/Thévenon 2013). Auch wenn viele familienpolitische Maßnahmen national einheitlich sind, existiert v.a. bei der Kinderbetreuung eine zum Teil erhebliche Variation innerhalb eines Landes. Die Befundlage zum Einfluss regionaler Unterschiede durch die Familienpolitik, insbesondere von Kinderbetreuung, auf die Fertilität ist heterogen. Einen positiven Effekt von Kindertagesstätten auf den Übergang zum ersten Kind zeigen Rindfuss et al. (2007) für Norwegen. Unterschiedlich sind die regional differenzierten Befunde zu Deutschland (Bauernschuster et al. 2013; Hank et al. 2004), wobei der Analysezeitraum bei Bauernschuster et al. die Kita-Expansion in Westdeutschland berücksichtigt. Für Spanien kann Baizan (2009) einen positiven Effekt von formaler Kinderbetreuung nachweisen, der beim Übergang zu höheren Paritäten bei einer höheren Frauenerwerbsquote verstärkt wird.

Regionale Arbeitsmärkte unterscheiden sich teilweise erheblich zwischen Kreisen, dies betrifft Arbeitslosenquote, Wirtschaftsstruktur und Frauenerwerbstätigkeit. Dabei ist der Einfluss von Arbeitslosigkeit eng mit dem Wohlstand einer Region verbunden. Die zunehmende berufliche und ökonomische Unsicherheit im frühen Lebensverlauf (Mills/Blossfeld 2005) ist bei hoher Arbeitslosigkeit und im Dienstleistungssektor besonders deutlich. Für beide Faktoren wurden für Norwegen negative Effekte auf die Fertilität gefunden (Kravda/ 2002), wobei weniger individuelle, als aggregierte regionale Effekte ausschlaggebend sind. Gemischte Befunde finden Hank und Huinink (2015) für Deutschland: Einen positiven Effekt der Arbeitslosigkeit auf die Kinderzahl und einen negativen auf die Intention einer Geburt in den nächsten zwei Jahren.

\section{Daten, Methoden und Forschungsdesign}

\subsection{Daten}

Die vorliegende Studie basiert auf den Mikrodaten der Haushaltebefragung des Zensus 2011 (Bechthold 2016). Circa 10 \% der deutschen Bevölkerung, rund 7,9 Mio. 
Menschen, wurden im Rahmen dieser Haushaltebefragung zu zusätzlichen soziodemografischen Informationen befragt, die nicht in den Registerdaten enthalten sind (Statistisches Bundesamt 2010). Die Ermittlung der Haushaltsstichprobe erfolgte nach einer regional geschichteten Zufallsauswahl von Anschriften aus dem Anschriften- und Gebäuderegister. Alle Personen einer ausgewählten Anschrift wurden befragt, wobei Auskunftspflicht bestand. Sensible Sonderbereiche, wie psychiatrische Einrichtungen oder Justizvollzugsanstalten, und im Ausland tätige Angehörige der Bundeswehr, der Polizeibehörden und des Auswärtigen Dienstes sowie ihre dort ansässigen Familien wurden vorab aus der Auswahlgrundlage entfernt, da das Bereitstellen von Informationen für diese Personengruppen mit sozialer Benachteiligung verbunden sein kann.

Die Haushalts- und Familienzusammenhänge aller an einer Anschrift lebenden Personen wurden nicht direkt erfragt, sondern nachträglich im Rahmen der Haushaltegenerierung erzeugt (Statistisches Bundesamt 2015; Kreuzmair/Reisch 2013). Hierbei wurden klassische Haushalts- und Familienzusammenhänge anhand von direkten Verzeigerungen der Melderegister, wie Ehen, eingetragene Lebenspartnerschaften oder Nachkommensbeziehungen, gebildet. Die Angabe der Nachkommensbeziehung ist üblicherweise bis zur Vollendung des 18. Lebensjahres des Kindes vorhanden. Verzeigerungen, die über dieses Lebensjahr hinaus im Register enthalten sind, wurden ebenfalls bei der Haushaltegenerierung berücksichtigt. Zur Durchführung der Zuordnung weiterer Lebensformen, wie beispielsweise nicht-eingetragener Partnerschaften, wurden weitere Registerinformationen, wie Zuzugsdaten oder Zuzugsanschriften, genutzt.

Die Mikrodaten des Zensus sind innerhalb eines relationalen Datenmodells strukturiert, bei dem alle Teildatensätze des Zensus als eigene Tabelle abgelegt sind und über Identifikatoren miteinander verknüpft werden können. Innerhalb der vorliegenden Studie wurden die Einzeldaten der Haushaltebefragung sowie die im Rahmen der Haushaltegenerierung nachträglich gebildeten Familienzusammenhänge nach nationaler Typisierung verwendet. Da die für die Berechnung benötigten Familieninformationen nicht für Personen aus kleineren Gemeinden vorliegen, wurden diese Personen aus der Analyse ausgeschlossen.

Zunächst wurden alle Frauen der Haushaltsstichprobe selektiert und anhand der generierten Zusammenhänge über Familientyp und Familiengröße für jede Frau die Anzahl der Kinder gebildet. ${ }^{4}$ Die Stichprobenergebnisse wurden mittels eines Hochrechnungsfaktors auf die Zensus-Gesamtbevölkerung hochgerechnet, um Vergleiche mit anderen Datenquellen zu ermöglichen. Zu berücksichtigen ist

4 Zu beachten ist, dass für die vorliegende Untersuchung das generative Verhalten von Frauen im Allgemeinen relevant ist und nicht auf der Ebene der Familien abgebildet ist. Daher werden Frauen in gleichgeschlechtlichen Lebensgemeinschaften als zwei Fälle erfasst. Ferner sei einschränkend erwähnt, dass es bei der Zuordnung von Müttern in Mehrgenerationenfamilien bedingt durch die Methodik der Haushaltegenerierung zu einer Untererfassung der Kinder kommen kann. Das Familienkonzept des Zensus 2011 bezieht sich auf direkte Beziehungen (ersten Grades) zwischen Vorfahren und Nachfahren, d.h. auf Beziehungen zwischen Eltern und Kindern. 
hierbei, dass der Wohnort der Frau zum Zeitpunkt des Zensusstichtages abgebildet wird und nicht zum Zeitpunkt der Geburt.

Zum Zeitpunkt der Zensusbefragung existierten 412 Kreise. Infolge der Gebietsreformen in Mecklenburg-Vorpommern 2011 wurden 18 Kreise in acht Kreise zusammengefasst. Alle Daten für diese Kreise wurden auf Basis von Kühntopf (2015) umgerechnet, so dass alle Ergebnisse - nach den Kreisgebieten mit dem Stand vom 1.1.2015 - für 402 Kreise angegeben werden.

\subsection{Berechnung des Multiplikators und Schätzung der CTFR}

Um die endgültige Kinderzahl pro Frau bzw. die CTFR zu schätzen, wird die durchschnittliche Anzahl der Kinder im Haushalt der Frauen verwendet. Die biologische Kinderzahl und damit auch die endgültige Kinderzahl sind im Zensus nicht enthalten. Die Kinderzahl im Haushalt beim Zensus unterschätzt die endgültige Kinderzahl v.a. aus zwei Gründen: Erstens werden bereits aus dem Haushalt ausgezogene Kinder nicht erfasst, wodurch insbesondere bei Frauen ab dem 38. Lebensjahr die erfasste Kinderzahl zunehmend die CTFR unterschätzt. Zweitens wird bei Frauenkohorten, die jünger als 45 Jahre alt sind, die endgültige Kinderzahl unterschätzt, da diese ihre Fertilitätsbiografie noch nicht abgeschlossen haben.

Zunächst werden die Jahrgänge identifiziert, bei denen die Verzerrungen zwischen der Kinderzahl im Haushalt und der CTFR am geringsten sind. In Abbildung 1 werden die Zensus-Werte und die MZ-Werte für die Kinderzahl im Haushalt mit der endgültigen Kinderzahl basierend auf der amtlichen Geburtenstatistik (1960-70) ${ }^{5}$ bzw. der linearen Trendextrapolation (1971-75) verglichen. ${ }^{6}$ Der Vergleich zeigt, dass für die 38- bis 42-jährigen Frauen, die Kohorten 1969-72, die Differenz mit 6,6 \% bis $7,8 \%$ am geringsten ist. Mit zunehmendem Abstand von diesen Jahrgängen vergrößert sich die Differenz zwischen den Kindern im Haushalt und der endgültigen Kinderzahl. Diese Unterschätzung ist zweifellos ein Nachteil. Da die Geburtenfrage im Zensus jedoch nicht gestellt wurde, ist dieses Vorgehen alternativlos. Um eine ausreichende Anzahl an Fällen für eine Kreisbetrachtung zu erhalten, werden die Zahlen der vier identifizierten Kohorten 1969-1972 zusammengenommen. Die Unterschätzung hält sich durch die Auswahl der Jahrgänge 1969-72 in Grenzen.

Die prozentuale Unterschätzung der CTFR beträgt 7,17 \% bei der Verwendung der Anzahl der Kinder im Haushalt für die Kohorten 1969-72 im Zensus. Es ist plausibel, dass sie für die meisten Kreise relativ gleichmäßig verteilt ist und dass Unterschiede v.a. mit einem jüngeren Geburtsalter und dem damit verbundenen früheren Auszug verbunden sind. Dies gilt v.a. für Ostdeutschland, wo das Erstgeburtsalter

5 Die Daten der amtlichen Geburtenstatistik berücksichtigen beim Jahrgang 1970 die altersspezifischen Fertilitätsraten (ASFR) bis einschließlich der ASFR 42. Die danach noch folgenden Geburten sind sehr gering, ihr Effekt summiert sich auf $<=0,01$.

6 Dabei sei auf erhebungsbedingte Unterschiede hingewiesen, da der Zensus - ähnlich wie der MZ und im Unterschied zur Geburtenstatistik - Kinder von zugewanderten Frauen berücksichtigt, die im Ausland geboren wurden (vgl. Pötzsch 2010). 
Abb. 1: Vergleich von durchschnittlicher Kinderzahl im Haushalt (Zensus und MZ) und endgültiger Kinderzahl (amtliche Geburtenstatistik und lineare Extrapolation) der Geburtskohorten 1960-75 in Deutschland

Durchschnittliche Kinderzahl je Frau

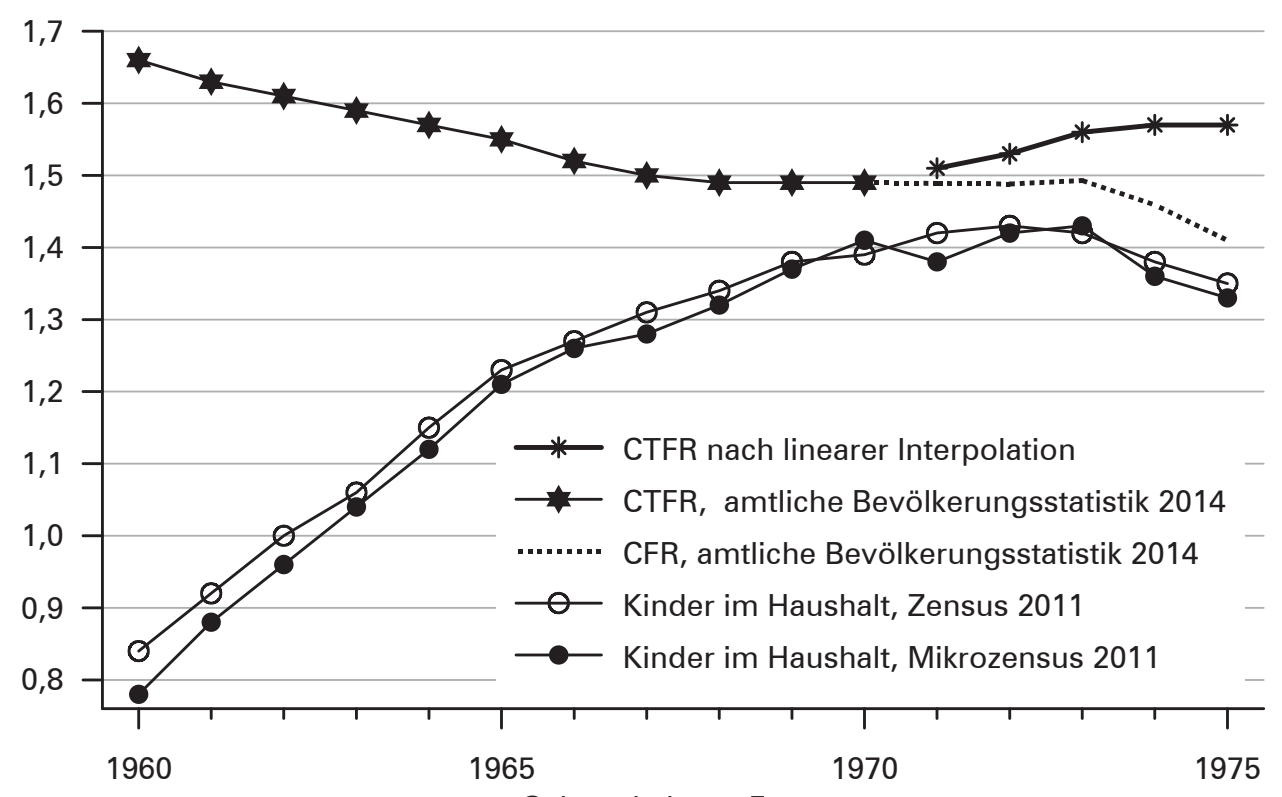

Geburtskohorte Frauen

Quelle: Eigene Berechnung basierend auf Zensus 2011 und MZ 2011; ferner Statistisches Bundesamt (2014); Goldstein/Kreyenfeld (2013).

erheblich geringer ist (Statistisches Bundesamt 2014), so dass bei mehr ostdeutschen Müttern der Jahrgänge 1969-72 im Jahr 2011 die Kinder bereits aus dem Elternhaus ausgezogen sind. Entsprechend beträgt die Unterschätzung in den alten Bundesländern nur 5,86 \%, während sie in den neuen mit 12,20\% deutlich höher ist (Tab. 1). Eine weitere Differenzierung nach Großstädten und anderen Regionen würde den Schätzwert weiter verbessern. Allerdings ist dies aufgrund der Datenlage nur begrenzt - bspw. für die Stadtstaaten - möglich. Zudem würde die Kompliziertheit des Verfahrens in einem schlechten Verhältnis zum Präzisionsgewinn stehen. Da die Kinderzahl im Haushalt für Ostdeutschland die CTFR weitaus stärker unterschätzt, wird ein zwischen West- und Ostdeutschland differenzierter Multiplikator angewendet. Mit diesen Multiplikatoren und den kreisspezifischen Werten zur durchschnittlichen Kinderzahl im Haushalt pro Frau im Zensus wird die CTFR der Frauenjahrgänge 1969-72 für alle 402 Kreise geschätzt. 
Tab. 1: Vergleich der durchschnittlichen Kinderzahl im Haushalt nach Zensus und CTFR

\begin{tabular}{ccccccccccc}
\hline Jahr- & \multicolumn{3}{c}{ CTFR (Geburtenstatistik) } & \multicolumn{3}{c}{ Zensus 2011} & \multicolumn{3}{c}{ Differenz (CTFR - Zensus) } \\
gang & Ost & West & gesamt & Ost & West & gesamt & Ost & West & gesamt & in \% \\
\hline 1960 & 1,80 & 1,60 & 1,66 & 0,56 & 0,91 & 0,84 & 1,23 & 0,69 & 0,82 & 97,3 \\
1961 & 1,76 & 1,58 & 1,63 & 0,63 & 0,99 & 0,92 & 1,13 & 0,59 & 0,71 & 77,6 \\
1962 & 1,72 & 1,56 & 1,61 & 0,69 & 1,08 & 1,00 & 1,03 & 0,48 & 0,61 & 61,1 \\
1963 & 1,68 & 1,54 & 1,59 & 0,75 & 1,14 & 1,06 & 0,93 & 0,40 & 0,52 & 49,3 \\
1964 & 1,64 & 1,53 & 1,57 & 0,86 & 1,22 & 1,15 & 0,78 & 0,30 & 0,41 & 35,9 \\
1965 & 1,60 & 1,52 & 1,55 & 0,94 & 1,30 & 1,23 & 0,67 & 0,22 & 0,33 & 26,5 \\
1966 & 1,56 & 1,50 & 1,52 & 1,04 & 1,32 & 1,27 & 0,52 & 0,17 & 0,25 & 19,8 \\
1967 & 1,55 & 1,47 & 1,50 & 1,13 & 1,35 & 1,31 & 0,42 & 0,12 & 0,19 & 14,2 \\
1968 & 1,51 & 1,47 & 1,49 & 1,20 & 1,37 & 1,34 & 0,31 & 0,10 & 0,15 & 11,1 \\
\hline 1969 & 1,49 & 1,48 & 1,49 & 1,28 & 1,40 & 1,38 & 0,21 & 0,07 & 0,11 & 7,8 \\
1970 & 1,46 & 1,49 & 1,49 & 1,29 & 1,42 & 1,39 & 0,17 & 0,07 & 0,10 & 7,2 \\
1971 & 1,50 & 1,51 & 1,51 & 1,37 & 1,43 & 1,42 & 0,13 & 0,09 & 0,09 & 6,6 \\
1972 & 1,51 & 1,53 & 1,53 & 1,38 & 1,44 & 1,43 & 0,14 & 0,10 & 0,10 & 7,1 \\
\hline 1973 & 1,53 & 1,57 & 1,56 & 1,38 & 1,43 & 1,42 & 0,15 & 0,14 & 0,14 & 9,8 \\
1974 & 1,54 & 1,57 & 1,57 & 1,35 & 1,39 & 1,38 & 0,19 & 0,19 & 0,18 & 13,3 \\
1975 & 1,54 & 1,58 & 1,57 & 1,33 & 1,35 & 1,35 & 0,21 & 0,23 & 0,22 & 16,6 \\
\hline
\end{tabular}

Anmerkungen: Differenzen sind rundungsbedingt. Die grau schattierten Jahrgänge werden in der Analyse berücksichtigt.

Quelle: Eigene Berechnung basierend auf Zensus 2011(hochgerechnete Werte) und amtlicher Geburtenstatistik (1960-70: Statistisches Bundesamt (2014); 1971-75: Goldstein/Kreyenfeld (2013).

\subsection{Sensitivitätsanalyse: Vergleich der Zensusbefunde mit dem Mikrozensus}

Im Folgenden werden die berechneten Werte zur durchschnittlichen Kinderzahl von Frauen im Haushalt (Familienebene) aus dem Zensus geprüft, indem sie mit denen des Mikrozensus (MZ) aus dem gleichen $\mathrm{Jahr}^{7}$ verglichen werden (siehe Tab. 2). Ein Vergleich mit dem MZ 2012 hätte zwar den Vorteil, dass dort die tatsächlichen Geburten erfragt wurden, allerdings sind bei den ausgewählten Kohorten die Fertilitätsbiografien noch nicht abgeschlossen. Insofern ist der Vergleich der ZensusErgebnisse mit dem MZ aus dem gleichen Jahr und mit vergleichbaren Variablen aussagekräftiger, um die Plausibilität der Zensus-Analysen zu überprüfen.

7 Beide Befragungen stammen aus dem Jahr 2011. Beim Zensus war der Stichtag 09.05.2011 relevant, während beim $M Z$ die Befragung - bedingt durch die unterjährige Erhebungsmethodik - zu unterschiedlichen Zeitpunkten stattgefunden hat. Bei den hier ausgewählten Kohorten wird davon ausgegangen, dass die dadurch entstandenen Monatsdifferenzen zu einer vernachlässigbaren Verzerrung führen. 
Tab. 2: Durchschnittliche Kinderzahl im Haushalt von Frauen: Vergleich Zensus und Mikrozensus

\begin{tabular}{rrrrrrrrrrr}
\hline & \multicolumn{3}{c}{ MZ 2011 } & \multicolumn{4}{c}{ Zensus 2011 } & \multicolumn{3}{c}{ Differenz (MZ - Zensus) } \\
& Ost & West & gesamt & Ost & West & gesamt & Ost & West & gesamt & in \% \\
\hline 1960 & 0,48 & 0,86 & 0,78 & 0,56 & 0,91 & 0,84 & $-0,08$ & $-0,05$ & $-0,06$ & $-7,1$ \\
1961 & 0,56 & 0,96 & 0,88 & 0,63 & 0,99 & 0,92 & $-0,07$ & $-0,03$ & $-0,04$ & $-4,3$ \\
1962 & 0,62 & 1,05 & 0,96 & 0,69 & 1,08 & 1,00 & $-0,07$ & $-0,03$ & $-0,04$ & $-4,0$ \\
1963 & 0,70 & 1,12 & 1,04 & 0,75 & 1,14 & 1,06 & $-0,05$ & $-0,02$ & $-0,02$ & $-1,9$ \\
1964 & 0,81 & 1,20 & 1,12 & 0,86 & 1,22 & 1,15 & $-0,05$ & $-0,02$ & $-0,03$ & $-2,6$ \\
1965 & 0,90 & 1,30 & 1,21 & 0,94 & 1,30 & 1,23 & $-0,04$ & 0,00 & $-0,02$ & $-1,6$ \\
1966 & 1,00 & 1,32 & 1,26 & 1,04 & 1,32 & 1,27 & $-0,04$ & 0,00 & $-0,01$ & $-0,8$ \\
1967 & 1,04 & 1,34 & 1,28 & 1,13 & 1,35 & 1,31 & $-0,09$ & $-0,01$ & $-0,03$ & $-2,3$ \\
1968 & 1,14 & 1,35 & 1,32 & 1,20 & 1,37 & 1,34 & $-0,06$ & $-0,02$ & $-0,02$ & $-1,5$ \\
1969 & 1,25 & 1,40 & 1,37 & 1,28 & 1,40 & 1,38 & $-0,03$ & $-0,00$ & $-0,01$ & $-0,7$ \\
1970 & 1,28 & 1,44 & 1,41 & 1,29 & 1,42 & 1,39 & $-0,01$ & 0,02 & 0,02 & 1,4 \\
1971 & 1,28 & 1,40 & 1,38 & 1,37 & 1,43 & 1,42 & $-0,09$ & $-0,03$ & $-0,04$ & $-2,8$ \\
1972 & 1,38 & 1,43 & 1,42 & 1,38 & 1,44 & 1,43 & $-0,00$ & $-0,01$ & $-0,01$ & $-0,7$ \\
1973 & 1,40 & 1,42 & 1,43 & 1,38 & 1,43 & 1,42 & $-0,02$ & $-0,01$ & 0,01 & 0,7 \\
1974 & 1,32 & 1,35 & 1,36 & 1,35 & 1,39 & 1,38 & $-0,03$ & $-0,04$ & $-0,02$ & $-1,4$ \\
1975 & 1,31 & 1,35 & 1,33 & 1,33 & 1,35 & 1,35 & $-0,02$ & 0,00 & $-0,02$ & $-1,5$ \\
\hline
\end{tabular}

Anmerkung: Die Differenz in Prozent ist der Quotient aus Gesamtdifferenz und ZensusGesamtwert.

Quelle: Zensus 2011, MZ 2011, eigene Berechnung und Darstellung.

Die Differenzen der durchschnittlichen Kinderzahl von Frauen für die Kohorten 1960-75 und für Ost- und Westdeutschland sind nur minimal. Sie liegen zwischen 0,00 und 0,09. Diese Genauigkeit bestätigt die in Abschnitt 3.1 beschriebene Selektion und Filterung. Sie ist nur möglich, weil die Fallzahlen in MZ und Zensus enorm hoch sind und die Befragung aufgrund des verpflichtenden Charakters wenig durch Non-Response-Probleme verzerrt wird.

\subsection{Forschungsdesign der multivariaten Analysen}

Die multivariaten und räumlichen Analysen dienen dem Ziel, einen breiten Überblick über regionale Faktoren der CTFR, ihr Zusammenspiel und die räumlichen Cluster zu generieren. Bei den linearen Regressionsanalysen fungieren die 402 Kreise als Fälle, wobei für Gesamtdeutschland sowie für West- und Ostdeutschland separate Analysen durchgeführt werden. Diese Differenzierung wird aus zwei Gründen vorgenommen: Zum einen weist die Literatur auf große demografische, kulturelle und strukturelle Unterschiede zwischen beiden Regionen hin (u.a. Goldstein/ Kreyenfeld 2011; Schneider et al. 2012), zum anderen zeigen eigene Voranalysen erhebliche Ost-West-Unterschiede. Diese Unterschiede lassen sich nicht durch einen West-Ost-Dummy sinnvoll erfassen, da ein solcher mit mehreren unabhängigen 
Variablen unterschiedlich interagiert. Die hier verwendete Kreisebene hat gewisse Limitationen, da wie bei allen Makroanalysen auf Kreisebene das "Modifiable Areal Unit Problem“ (MAUP) (Openshaw 1984) auftritt, das bei multivariaten Analysen zu Interpretationsproblemen führen kann (Fotheringham/Wong 1991).

Abhängige Variable ist die in dieser Studie berechnete kreisspezifische CTFR für Frauen der Jahrgänge 1969-72. Die Datengrundlage des Zensus beinhaltet 193.934 Frauen der Kohorten 1969-72, die alle in die Berechnung der 402 kreisspezifischen CTFR-Werte eingegangen sind.

Die unabhängigen Variablen basieren auf kreisspezifischen Aggregierungen der Mikrodaten der Haushaltsstichprobe des Zensus 2011 (Kompositionsvariablen) sowie auf der INKAR-Datenbank (regionale Opportunitäten; INKAR 2015). Die Zensus-Aggregierungen werden für dieselben Kohorten (1969-72) wie die abhängige Variable berechnet und der Kreisumschätzer für die Gebietsreform in MecklenburgVorpommern angewendet. Folgende Anteile werden als unabhängige Kompositionsvariablen verwendet: Migrationshintergrund, niedriger Bildungsabschluss (ISCED 1-2), hoher Bildungsabschluss (ISCED 5A, 6), Katholiken, evangelische Christen, verheiratete Frauen, Frauen in nichtehelichen Lebensgemeinschaften (NEL) sowie die Summe der Frauen, die in Ehe oder in NEL leben.

Zur Abbildung der regionalen Opportunitäten werden die Durchschnittswerte von 1995-2011 (sofern vorhanden) ${ }^{8}$ verwendet, da der Einfluss dieser regionalen Faktoren in der Phase der generativen Entscheidung im Lebenslauf maßgeblich ist. Diese Phase umfasst bei den Frauen, die im Jahr 2011 um die 40 Jahre alt sind, den Zeitraum der zurückliegenden zehn oder mehr Jahre. Folgende Variablen werden als regionale Opportunitäten verwendet: Beschäftigtenquote im tertiären Sektor, Arbeitslosenquote (als Anteil an der gesamten Erwerbsbevölkerung), Beschäftigtenquote in Forschung und Entwicklung (FuE), Anteil an Frauen in Teilzeitarbeit, Beschäftigtenquote der Frauen, Erwerbsquote der Frauen, Bruttoinlandsprodukt je Einwohner, Bruttolohn und -gehalt, Anteil an Kinderarmut, Betreuungsquote von Kleinkindern von null bis zwei Jahren, Betreuungsquote von Vorschulkindern zwischen drei und fünf Jahren, Ganztags-Betreuungsquote von Vorschulkindern, ${ }^{9}$ pädagogisches Personal in Tageseinrichtungen in Relation zur Kinderzahl, Anteil von Fünf- und mehr Raum-Wohnungen, Wohnfläche pro Person in Quadratmetern, naturnähere Fläche je Einwohner und die Geschlechterproportion der 20- bis 39-Jährigen (Anteil von Frauen/Männern). Der natürliche Logarithmus (zur Basis e) der Bevölkerungsdichte wird zur Operationalisierung des Effektes des Urbanisierungsgrades verwendet.

8 Bei den meisten INKAR-Variablen (BBSR 2015) sind Werte von 1995-2011 vorhanden. Bei folgenden Variablen geht die Datenreihe nicht so weit zurück: Arbeitslosenquote ab 1998, Bruttogehalt ab 2000, FuE-Beschäftigungsquote ab 2003, familienpolitische Betreuungsquoten ab 2007, Beschäftigtenquote im tertiären Sektor und Frauenerwerbsquote ab 2008. Dies ist ein Nachteil, jedoch kann aufgrund der Entwicklung bei den anderen Variablen davon ausgegangen werden, dass die Relation dieser Variablen zwischen den Kreisen im gesamten Zeitraum relativ ähnlich war.

9 Dieser Indikator misst den Anteil der Kinder von drei bis fünf Jahren in Kitas mit einer Betreuungszeit von sieben und mehr Stunden pro Tag. 


\section{$4 \quad$ Ergebnisse}

\subsection{CTFR auf Kreisebene}

Die Ergebnisse für die geschätzte durchschnittliche CTFR der Kohorten 1969-72 für alle 402 Kreise werden in Abbildung 2 grafisch dargestellt. Die einzelnen Werte für jeden Kreis sind in Tabelle A-1 im Anhang sowie in der Excel-Tabelle im OnlineAnhang abgetragen. Die Spannbreite liegt zwischen 1,05 in Passau und 2,01 in Cloppenburg (siehe Abb. 3). In 28 Kreisen ist die CTFR unterhalb von 1,3, insbesondere in Großstädten wie Köln, Hamburg, Stuttgart oder München. In 44 Kreisen liegt sie oberhalb von 1,7. Viele dieser Kreise mit einer relativ hohen Kohortenfertilität liegen im Allgäu, in der Odenwaldregion, in Südfranken, in Ostsachsen und in der Region zwischen Münster und dem Emsland.

Bezüglich des kreisspezifischen Einflusses auf die endgültige Kinderzahl von Frauen ist die geschätzte CTFR aussagekräftiger und besser interpretierbar als die TFR. Aufgrund fehlender regionaler Daten zur CTFR wird manchmal für solche Fragestellungen die TFR herangezogen, jedoch oft missinterpretiert. Denn es gibt fundamentale Unterschiede zwischen beiden Messkonzepten, die sich anhand eines Lexis-Diagramms zu Alter und Zeit illustrieren lassen (Luy 2010). Die TFR zeigt nicht nur das Quantum, sondern auch einen Timingeffekt an (Bongaarts/Feeney 1998). Auch die Binnenwanderung von Müttern beeinflusst beide Indikatoren in vielen Kreisen unterschiedlich. Dies wird im Folgenden anhand des Vergleichs der CTFR mit vorhandenen TFR-Daten auf Kreisebene gezeigt.

Datengrundlage für die TFR-Werte sind Schätzungen des BBSR (2015). Für den Vergleich wird die TFR der Jahre verwendet, in denen das durchschnittliche Alter der Mütter bei der Geburt (MAC) der CTFR-Kohorten 1969-72 lag. Im Jahr 1999 lag das MAC bei 30,1 Jahren, also etwa im Alter der 1969 geborenen Frauen (Statistisches Bundesamt 2012). Das MAC ist bis 2003 auf 30,67 Jahre angestiegen, was dem 1972er Jahrgang entspricht. Entsprechend wird für die Kreise der Durchschnitt der TFR-Werte der Jahre 1999-2003 für den Vergleich mit den CTFR-Werten der Kohorten 1969-72 verwendet. Die Verwendung der Fünf-Jahre-Durchschnitte hat auch den Vorteil, dass TFR-Schwankungen auf Kreisebene geglättet werden. Grundsätzlich ist der Vergleich von CTFR und TFR problematisch. Hier geht es darum zu zeigen, dass und warum die hier gewonnenen CTFR-Werte erheblich von den bisher bekannten TFR-Werten divergieren.

Der Zusammenhang zwischen beiden Fertilitätsindikatoren auf Kreisebene liegt bei einem Pearson'schen Korrelationskoeffizienten von 0,56. Die hohe Abweichung von 1 verdeutlicht, dass es einen großen Unterschied macht, ob man ein Kohortenmaß (CTFR) oder ein Periodenmaß (TFR) betrachtet. Durchschnittlich ist im Vergleich der CTFR (1969-72) mit der TFR (1999-2003) die CTFR um 0,12 höher, entsprechend unterschätzt daher die TFR die tatsächlichen Geburten pro Frau. Dies ist bei Fertilitätsdaten auf Kreisebene aus zwei Gründen umso problematischer: Erstens, wenn der Aufschub der Geburten in einem unterschiedlichem Tempo erfolgt (und damit die Verzerrung durch den Timingeffekt divergiert) und zweitens aufgrund von Binnenwanderung zwischen verschiedenen Kreisen. Beides hat auf die TFR der 
Abb. 2: Endgültige Kinderzahl (CTFR) auf Kreisebene: Schätzwerte der Geburtsjahrgänge 1969-72

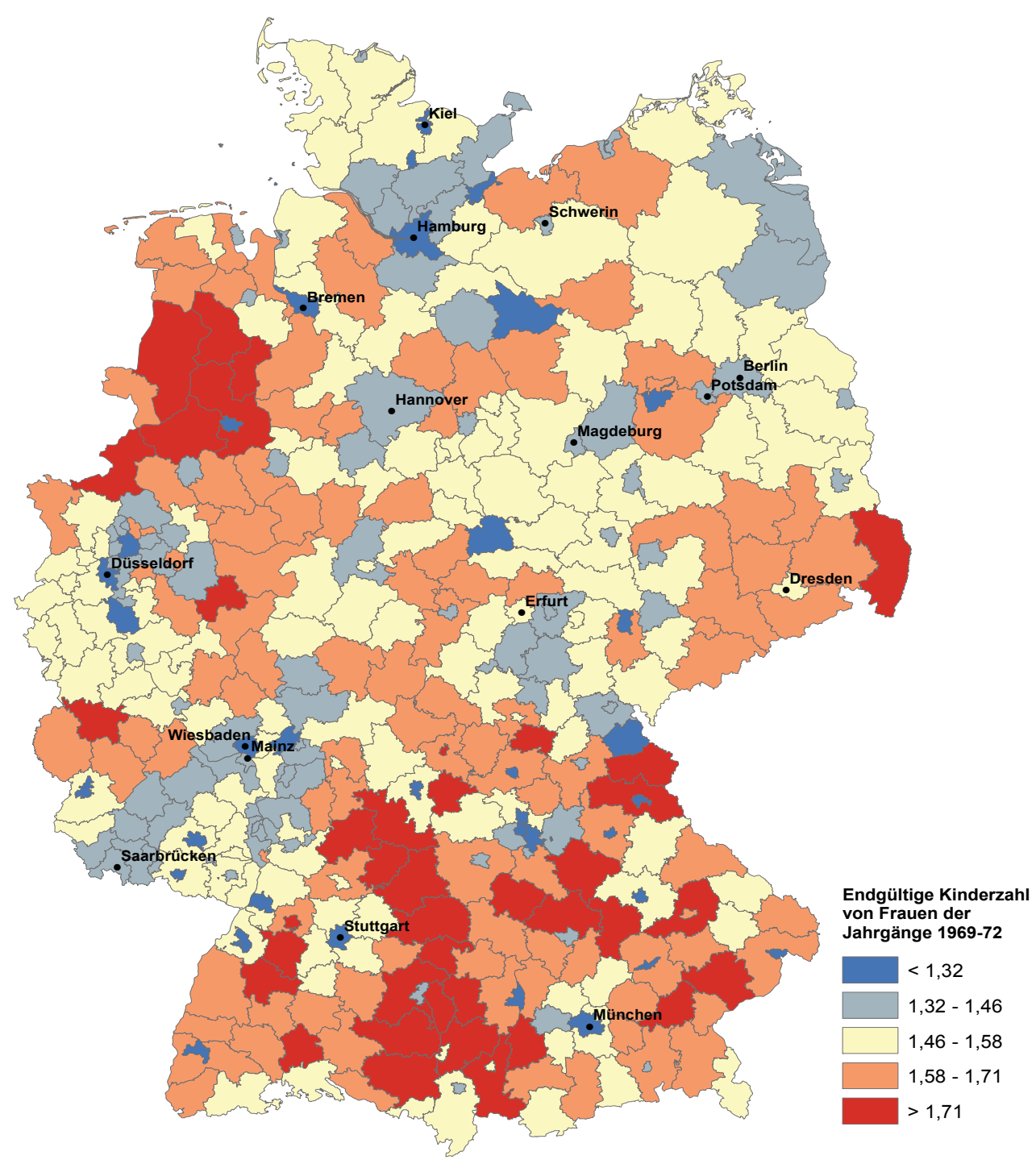

Anmerkungen: Eine Karte mit nummeriertem Schlüssel und eine Liste der Kreisnamen sowie die zugehörigen CTFR-Werte werden im Anhang zur Verfügung gestellt. Ferner kann ein Excel-Dokument mit den kreispezifischen CTFR unter folgendem Link heruntergeladen werden: http://www.comparativepopulationstudies.de/index.php/CPoS/article/view/278/251.

Quelle: Eigene Berechnung basierend auf Zensus 2011. Kartengrundlage: (c) GeoBasisDE/BKG (2015). 
Abb. 3: Geschätzte endgültige Kinderzahl (CTFR) auf Kreisebene: Top 10 und Bottom 10

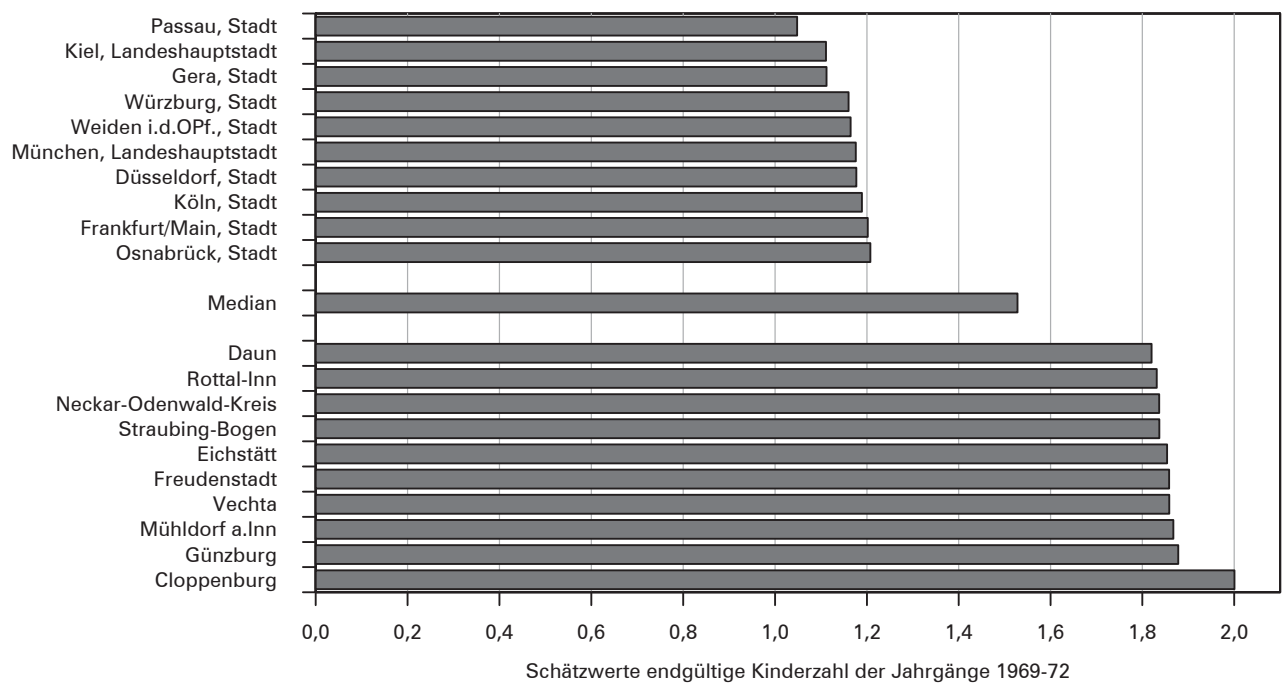

Quelle: Eigene Berechnung basierend auf Zensus 2011.

Kreise in Deutschland eine erhebliche Wirkung. Die Frage, wie viele Kinder Frauen in bestimmten Kreisen haben (oder bekommen), lässt sich inhaltlich mit den TFR auf Kreisebene nicht befriedigend beantworten. Demgegenüber steht der Vorteil der TFR, dass sie eine hohe Aktualität ermöglicht.

Die Daten zur endgültigen Kinderzahl dagegen ermöglichen eine Antwort auf die Frage, wie viele Kinder Frauen einer Kohorte haben. Temporär kinderlose Frauen, die für die Ausbildungsphase oder das Studium in eine andere Stadt umziehen, beeinflussen die TFR dieser Stadt negativ, denn sie werden im Nenner mitgezählt und führen ggf. zu einer erheblichen Unterschätzung der Fertilität von Frauen in Universitätsstädten. Diese Phänomene führen dazu, dass die Abweichung von CTFR und TFR auf Kreisebene zwischen +0,50 im Brandenburger Kreis Elbe-Elster und -0,30 in Lüchow-Dannenberg (Niedersachsen) liegt, also eine Spannbreite von über 0,8. Die kreisspezifische Variation der TFR-Verzerrung im Vergleich zur CTFR ist erheblich, wobei drei Phänomene zentral sind:

1. In Ostdeutschland ist die Timing-Komponente der TFR besonders deutlich, da der Aufschub des MAC nach der Wende enorm hoch war. Entsprechend ist die CTFR in fast allen ostdeutschen Kreisen um mindestens 0,2 höher als die TFR - in 37,7\% sogar mehr als 0,3. Gera ist der einzige von 77 ostdeutschen Kreisen, in dem die TFR höher ist.

2. In mehreren Kreisen sind Stadt-Land-Wanderungen nach der Geburt von Kindern sichtbar, wobei Familien mit kleinen Kindern von der Stadt in angrenzende ländliche Kreise ziehen. Dieses Phänomen zeigt sich in Köln, Düsseldorf, Essen und Frankfurt am Main und besonders deutlich in Bayern, wo in München, Ingolstadt, Amberg, Weiden, Nürnberg, Fürth und Erlangen mehrere Städte eine niedrigere CTFR im Vergleich zur TFR haben. 
3. In mehreren Studentenstädten wie Münster $(0,33)$, Tübingen $(0,23)$ oder Heidelberg $(0,48)$ ist die CTFR erheblich höher als die TFR, die durch die überwiegend temporär dort lebenden und in dieser Zeit gering fertilen Studentinnen gedrückt wird.

\subsection{Makroanalysen zu regionalen Faktoren der CTFR}

Auf Basis der neu gewonnenen Daten zur Kohortenfertilität auf Kreisebene lassen sich regionale Faktoren auf der Makroebene analysieren. Tabelle 3 zeigt die Zusammenhänge zwischen der CTFR und einigen sozialstrukturellen, ökonomischen, politischen und geografischen Faktoren, wobei zwischen West- und Ostdeutschland differenziert wird.

Bei den sozialstrukturellen Faktoren gibt es deutliche Unterschiede zwischen West- und Ostdeutschland. Bspw. ist die negative Korrelation der Arbeitslosenquote und der NEL jeweils mit der CTFR nur in Westdeutschland signifikant. Bemerkenswert ist die Religionsvariable: Während ein hoher Katholikenanteil nur in Westdeutschland signifikant mit höherer Kohortenfertilität assoziiert ist, verbirgt sich hinter dem negativen Vorzeichen beim Anteil evangelischer Christen für Gesamtdeutschland ein signifikant positiver Zusammenhang für Ostdeutschland. Die Kinderbetreuungsquoten sind nur in Westdeutschland signifikant. Der Zusammenhang der CTFR mit Kleinkindbetreuung und Ganztagsplätzen ist negativ, was erklärungsbedürftig ist. Da beide Variablen mit Arbeitslosigkeit korrelieren $(r=0,71$ und 0,67 für Gesamtdeutschland), könnte sich dahinter ein Drittvariableneffekt verbergen. Zudem sind sie aufgrund des betrachteten Zeitraums nur begrenzt aussagekräftig für die Fertilität der untersuchten Frauenkohorten (siehe 3.4).

Da Urbanisierung in der Literatur als ein zentraler Faktor der regionalen Fertilitätsunterschiede genannt wird (siehe 2.2), wird der Zusammenhang zwischen Bevölkerungsdichte und Kinderzahl pro Frau grafisch dargestellt (Abb. 4). Aufgrund der schiefen Verteilung der Bevölkerungsdichte wird diese Variable logarithmiert. Der Zusammenhang zwischen beiden Variablen ist deutlich negativ, der Korrelationskoeffizient Pearsons $r$ beträgt 0,54.

Im Folgenden werden die Ergebnisse linearer Regressionsanalysen für Gesamtdeutschland gezeigt, wobei die 402 Kreise die Fälle darstellen (siehe Tab. 4). ${ }^{10}$ In Modell 1 fließen lediglich die Kompositionsvariablen ein, in Modell 2 und 3 werden die von der Sozialstruktur beeinflussten Opportunitätsvariablen inkludiert und Modell 4 berücksichtigt die Variablen zu regionalen Opportunitäten. Modell 5 ist das Best-Fit-Modell, wenn man sich auf die sechs einflussreichsten Variablen fokussiert. Der negative Effekt der Beschäftigtenquote im tertiären Sektor, der Bevölkerungsdichte und einer Geschlechterrelation mit wenigen Männern ist über alle Modelle hinweg robust und hochsignifikant $(p<0,001)$. Ebenfalls als signifikant erweisen sich

10 Um Multikollinearität zu vermeiden, werden Variablen, deren Interkorrelationen über 0,7 liegen, nicht zusammen in einem Regressionsmodell verwendet. Daher wird die Variable Ganztagsbetreuungsquote der Drei- bis Fünf-Jährigen statt der Betreuungsquote für unter Dreijährige sowie bei den Wohlstandsindikatoren das BIP anstelle von Kinderarmut und Gehalt benutzt. 
Tab. 3: Bivariate Korrelationen auf Kreisebene zwischen der CTFR und ausgewählten Kompositions- und Opportunitätseffekten

\begin{tabular}{|c|c|c|c|c|}
\hline & Faktoren/Indikatoren & $\mathrm{D}$ & West & Ost \\
\hline \multirow{12}{*}{ 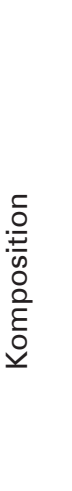 } & Bildung & & & \\
\hline & Anteil niedriger Bildungsabschluss (ISCED 1-2) & $-0,05$ & $-0,12^{*}$ & $-0,19$ \\
\hline & Anteil hoher Bildungsabschluss (ISCED 5A, 6) & $-0,49 * * *$ & $-0,52 * * *$ & $-0,33^{* *}$ \\
\hline & Migration & & & \\
\hline & Anteil Migrationshintergrund & $-0,20 * * *$ & $-0,31 * * *$ & $-0,32 * *$ \\
\hline & Religion & & & \\
\hline & Anteil Katholiken & $0,30 * * *$ & $0,33 * * *$ & 0,18 \\
\hline & Anteil evangelische Christen & $-0,03$ & $-0,10$ & $0,38 * * *$ \\
\hline & Lebensform & & & \\
\hline & Anteil verheirateter Frauen & $0,64 * * *$ & $0,73 * * *$ & $0,66 * * *$ \\
\hline & Anteil Frauen in NEL & $-0,23^{* * *}$ & $-0,31 * * *$ & $-0,11$ \\
\hline & Anteil Frauen verheiratet oder NEL & $0,67 * * *$ & $0,68 * * *$ & $0,67 * * *$ \\
\hline & Arbeitsmarkt & & & \\
\hline \multirow{13}{*}{ 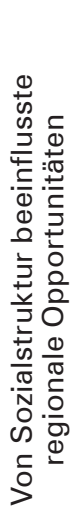 } & Beschäftigtenquote tertiärer Sektor & $-0,56 * * *$ & $-0,57 * * *$ & $-0,49 * * *$ \\
\hline & Arbeitslosenquote & $-0,28 * * *$ & $-0,42 * * *$ & $-0,01$ \\
\hline & Anteil FuE Beschäftigte & $-0,03$ & $-0,05$ & $-0,02$ \\
\hline & Frauenerwerbstätigkeit & & & \\
\hline & Anteil Frauen in Teilzeit & $-0,44^{* * *}$ & $-0,47 * * *$ & $-0,29 *$ \\
\hline & Beschäftigtenquote Frauen & $-0,02$ & 0,01 & 0,07 \\
\hline & Erwerbsquote Frauen & $0,21 * * *$ & $0,31 * * *$ & $0,29 *$ \\
\hline & Wohlstand & & & \\
\hline & Bruttoinlandsprodukt je Einwohner & $-0,36 * * *$ & $-0,41 * * *$ & $-0,45^{* * *}$ \\
\hline & Bruttolohn- und gehalt & $-0,14^{* *}$ & $-0,23^{* * *}$ & $-0,33^{* *}$ \\
\hline & Anteil Kinderarmut & $-0,46 * * *$ & $-0,50 * * *$ & $-0,41 * * *$ \\
\hline & Urbanisierung & & & \\
\hline & Bevölkerungsdichte logarithmiert & $-0,54 * * *$ & $-0,60 * * *$ & $-0,44^{* * *}$ \\
\hline \multirow{13}{*}{ 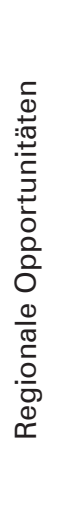 } & Städtischer/ländlicher Raum (Dummy) & $0,27^{* * *}$ & $0,30 * * *$ & $0,26^{*}$ \\
\hline & Familienpolitik & & & \\
\hline & Betreuungsquote Kleinkinder & $-0,20 * * *$ & $-0,38 * * *$ & $-0,29 *$ \\
\hline & Betreuungsquote Vorschulkinder & $-0,04$ & $-0,01$ & $-0,19$ \\
\hline & Ganztags-Betreuungsquote Vorschulkinder & $-0,33^{* * *}$ & $-0,55^{* * *}$ & $-0,16$ \\
\hline & Pädagogisches Personal in Tageseinrichtungen & $0,30 * * *$ & $0,47 * * *$ & $0,40 * * *$ \\
\hline & Wohnraum & & & \\
\hline & Anteil Fünf- und mehr Raum-Wohnungen & $0,59 * * *$ & $0,65^{* * *}$ & $0,48 * * *$ \\
\hline & Wohnfläche & $0,21 * * *$ & $0,23 * * *$ & $-0,02$ \\
\hline & Naturnähere Fläche je Einwohner & $0,12^{*}$ & 0,11 & $0,33^{* *}$ \\
\hline & Geschlechterproportionen & & & \\
\hline & Geschlechterproportion 20-39 & $-0,18^{* * *}$ & $-0,31 * * *$ & $-0,33^{* *}$ \\
\hline & $\mathrm{N}$ & 402 & 325 & 77 \\
\hline
\end{tabular}

Anmerkung: ${ }^{* *} \mathrm{p}<0.001 ;{ }^{* *} \mathrm{p}<0.01 ;{ }^{*} \mathrm{p}<0,05$.

Quelle: Eigene Berechnung basierend auf Zensus 2011 und BBSR (2015). 
Abb. 4: Zusammenhang Kinderzahl pro Frau und Einwohnerdichte in 402 Kreisen Deutschlands

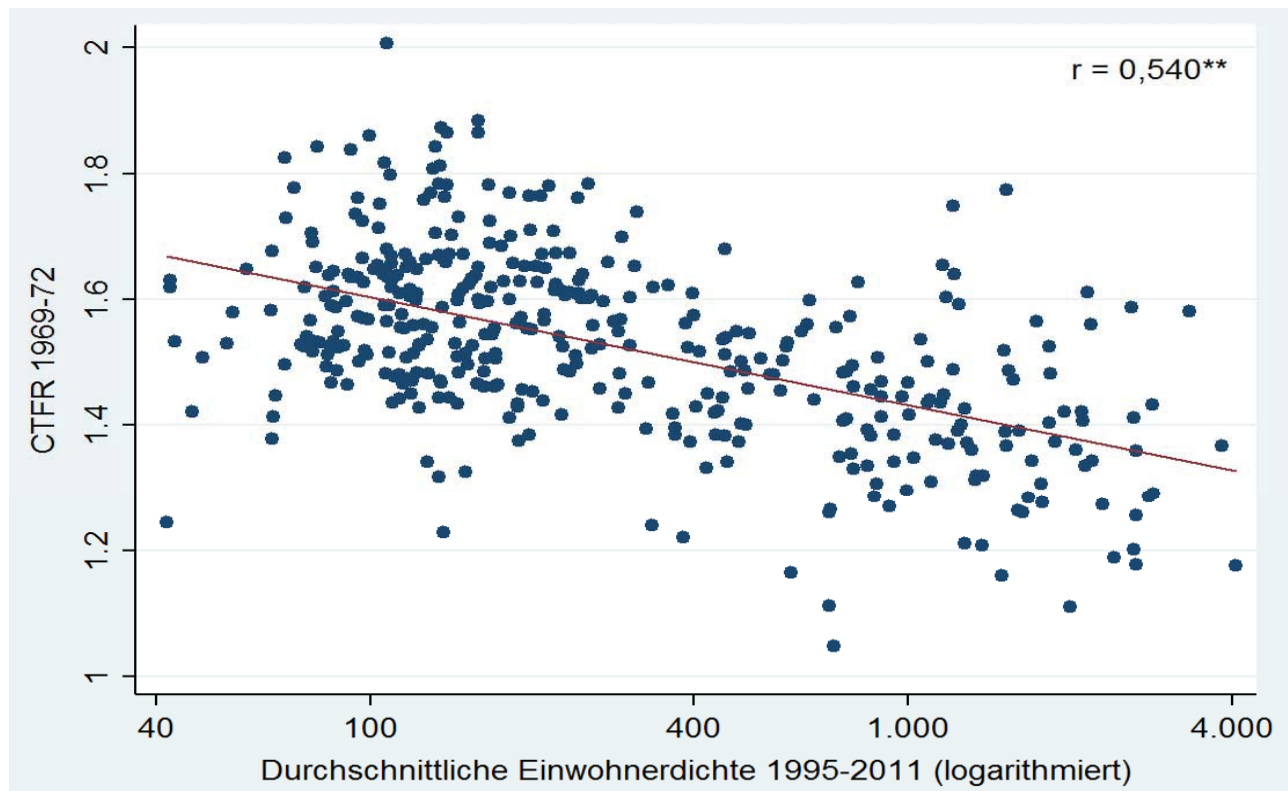

Quelle: Eigene Berechnung basierend auf Zensus 2011 und BBSR (2015).

die Katholikenquote und der Anteil an Fünf- und mehr Raum-Wohnungen, wobei das Signifikanzniveau zwischen den Modellen variiert. Bspw. reduziert sich bei der Katholikenquote der t-Wert von 7,1 in Modell 1 auf 2,2 in Modell 5. Der Migrantinnenanteil hat im Modell 1 einen signifikant negativen Effekt, wobei sich durch Inklusion der Variablen tertiärer Sektor und Bevölkerungsdichte (Modelle 3-5) das Vorzeichen umdreht - ebenfalls auf einem signifikanten Niveau. Da Migranten eher in Städten wohnen, ist der negative Zusammenhang in Modell 1 ein ökologischer Fehlschluss; er ist durch einen überlagernden Effekt der Urbanisierung verursacht. Modell 5 kommt mit diesen sechs Faktoren auf einen korrigierten Determinationskoeffizienten von 0,47. Dagegen verlieren die Effekte von Arbeitslosenquote, Teilzeitarbeit und Akademiker-Anteil in den Modellen ihre Signifikanz, in denen Bevölkerungsdichte und Wohnraum inkludiert sind (Modell 4).

Bei den Regressionsanalysen für Westdeutschland muss der Aufbau der Modelle aufgrund von Multikollinearität teilweise etwas abweichend erfolgen. ${ }^{11}$ Die Modelle zeigen, dass sich Kompositionseffekte und regionale Opportunitäten in ihrer Erklärungskraft ergänzen (Tab. 5). Das Best-fit-Modell W3 kommt auf eine Erklärungskraft von 0,51. Demzufolge ist die CTFR in den Kreisen höher, in denen wenige

11 Aufgrund von zu hohen Interkorrelationen sind das BIP, der Anteil an Fünf- und mehr RaumWohnungen und die Wohnfläche nicht berücksichtigt. Auch werden die Variablen Migrantenanteil und Bevölkerungsdichte nicht im gleichen Modell inkludiert. 
Tab. 4: OLS-Regressionen zur CTFR auf Kreisebene: Deutschland gesamt

\begin{tabular}{|c|c|c|c|c|c|}
\hline & Modell 1 & Modell 2 & Modell 3 & Modell 4 & Modell 5 \\
\hline $\begin{array}{l}\text { Anteil hoher } \\
\text { Bildungsabschluss }\end{array}$ & $\begin{array}{l}-0,0081^{* * *} \\
(0,0009)\end{array}$ & $\begin{array}{l}-0,0038^{* * *} \\
(0,0011)\end{array}$ & $\begin{array}{l}-0,0015 \\
(0,0011)\end{array}$ & $\begin{array}{l}0,0005 \\
(0,0013)\end{array}$ & \\
\hline $\begin{array}{l}\text { Anteil Migrations- } \\
\text { hintergrund }\end{array}$ & $\begin{array}{l}-0,0018^{* *} \\
(0,0006)\end{array}$ & $\begin{array}{l}-0,0011 \\
(0,0007)\end{array}$ & $\begin{array}{l}0,0024^{* *} \\
(0,0009)\end{array}$ & $\begin{array}{c}0,0022^{*} \\
(0,0009)\end{array}$ & $\begin{array}{l}0,0021^{* *} \\
(0,0008)\end{array}$ \\
\hline Anteil Katholiken & $\begin{array}{l}0,0019^{* * *} \\
(0,0003)\end{array}$ & $\begin{array}{c}0,00070^{*} \\
(0,00032)\end{array}$ & $\begin{array}{l}0,00085^{* *} \\
(0,00031)\end{array}$ & $\begin{array}{l}0,00073^{*} \\
(0,00031)\end{array}$ & $\begin{array}{r}0,00065^{*} \\
(0,00030)\end{array}$ \\
\hline $\begin{array}{l}\text { Beschäftigte tertiärer } \\
\text { Sektor }\end{array}$ & & $\begin{array}{l}-0,0037^{* * *} \\
(0,0007)\end{array}$ & $\begin{array}{l}-0,0024^{* * *} \\
(0,0007)\end{array}$ & $\begin{array}{l}-0,0026^{* * *} \\
(0,0007)\end{array}$ & $\begin{array}{l}-0,0026^{* * *} \\
(0,0007)\end{array}$ \\
\hline Arbeitslosenquote & & $\begin{array}{l}-0,0060^{* *} \\
(0,0020)\end{array}$ & $\begin{array}{l}-0,0060^{*} \\
(0,0024)\end{array}$ & $\begin{array}{l}-0,0005 \\
(0,0030)\end{array}$ & \\
\hline $\begin{array}{l}\text { Anteil Frauen in } \\
\text { Teilzeit }\end{array}$ & & $\begin{array}{l}-0,0045^{*} \\
(0,0020)\end{array}$ & $\begin{array}{l}-0,0029 \\
(0,0020)\end{array}$ & $\begin{array}{l}-0,0025 \\
(0,0019)\end{array}$ & \\
\hline BIP & & $\begin{array}{l}-0,0012 \\
(0,0011)\end{array}$ & $\begin{array}{l}-0,0017 \\
(0,0011)\end{array}$ & $\begin{array}{l}-0,0017 \\
(0,0010)\end{array}$ & \\
\hline $\begin{array}{l}\text { Bevölkerungsdichte } \\
\text { logarithmiert }\end{array}$ & & & $\begin{array}{l}-0,046^{* * *} \\
(0,010)\end{array}$ & $\begin{array}{l}-0,030^{* *} \\
(0,011)\end{array}$ & $\begin{array}{l}-0,038^{* * *} \\
(0,011)\end{array}$ \\
\hline $\begin{array}{l}\text { Geschlechterproportion } \\
20-39\end{array}$ & & & $\begin{array}{l}-0,61 * * * \\
(0,17)\end{array}$ & $\begin{array}{l}-0,74^{* * *} \\
(0,18)\end{array}$ & $\begin{array}{l}-0,65^{* * *} \\
(0,15)\end{array}$ \\
\hline $\begin{array}{l}\text { Ganztags-Betreuungs- } \\
\text { quote Vorschulkinder }\end{array}$ & & & & $\begin{array}{l}-0,00082^{*} \\
(0,00041)\end{array}$ & \\
\hline $\begin{array}{l}\text { Anteil Fünf- und mehr } \\
\text { Raum-Wohnungen }\end{array}$ & & & & $\begin{array}{c}0,0025^{*} \\
(0,0010)\end{array}$ & $\begin{array}{l}0,0035^{* * *} \\
(0,0008)\end{array}$ \\
\hline Konstante & $\begin{array}{l}1,616 * * * \\
(0,016)\end{array}$ & $\begin{array}{l}1,960^{* * *} \\
(0,045)\end{array}$ & $\begin{array}{l}2,595^{* * *} \\
(0,165)\end{array}$ & $\begin{array}{l}2,477^{* * *} \\
(0,194)\end{array}$ & $\begin{array}{l}2,311^{* * *} \\
(0,129)\end{array}$ \\
\hline $\mathrm{R}^{2}$ & $0,326 * * *$ & $0,433^{* * *}$ & $0,483^{* * *}$ & $0,500 * * *$ & $0,479 * * *$ \\
\hline Adj. $R^{2}$ & $0,321 * * *$ & $0,423^{* * *}$ & $0,471 * * *$ & $0,486 * * *$ & $0,472^{* * *}$ \\
\hline $\mathrm{N}$ & 402 & 402 & 402 & 402 & 402 \\
\hline
\end{tabular}

Anmerkungen: Unter den Koeffizienten steht in Klammern der Standardfehler.

*** $\mathrm{p}<0.001$; ** $\mathrm{p}<0.01$; ${ }^{*} \mathrm{p}<0,05$.

Quelle: Eigene Berechnung basierend auf Zensus 2011 und BBSR (2015).

Akademiker und viele Katholiken leben (Kompositionseffekt) sowie eine geringe Bevölkerungsdichte, relativ viele Männer, eine traditionellere Wirtschaftsstruktur und geringe Arbeitslosigkeit (regionale Opportunitäten) zusammenkommen. Abgesehen vom Effekt der Geschlechterproportionen sind alle diese Variablen auch in modifizierten Modellen (nicht gezeigt) robust signifikant. 
Tab. 5: OLS-Regressionen zur CTFR auf Kreisebene: Westdeutschland

\begin{tabular}{|c|c|c|c|c|c|}
\hline & Modell W1 & Modell W2 & Modell W3 & Modell W4 & Modell W5 \\
\hline $\begin{array}{l}\text { Anteil hoher } \\
\text { Bildungsabschluss }\end{array}$ & $\begin{array}{l}-0,0092^{* * *} \\
(0,0010)\end{array}$ & $\begin{array}{l}-0,0097^{* * *} \\
(0,0012)\end{array}$ & $\begin{array}{l}-0,0062 * * * \\
(0,0014)\end{array}$ & $\begin{array}{l}-0,0051 * * * \\
(0,0014)\end{array}$ & $\begin{array}{l}-0,0034^{* *} \\
(0,0011)\end{array}$ \\
\hline $\begin{array}{l}\text { Anteil Migrations- } \\
\text { hintergrund }\end{array}$ & $\begin{array}{l}-0,0013 \\
(0,0008)\end{array}$ & $\begin{array}{r}-0,00003 \\
(0,0007)\end{array}$ & & & \\
\hline Anteil Katholiken & $\begin{array}{l}0,0020^{* * *} \\
(0,0003)\end{array}$ & $\begin{array}{c}0,0004 \\
(0,0024)\end{array}$ & $\begin{array}{c}0,0005 \\
(0,0003)\end{array}$ & $\begin{array}{c}0,0007^{*} \\
(0,0003)\end{array}$ & $\begin{array}{l}0,0011^{* * *} \\
(0,0003)\end{array}$ \\
\hline $\begin{array}{l}\text { Beschäftigte } \\
\text { tertiärer Sektor }\end{array}$ & & $\begin{array}{l}-0,0024^{* *} \\
(0,0008)\end{array}$ & $\begin{array}{l}-0,0023^{* *} \\
(0,0008)\end{array}$ & $\begin{array}{l}-0,0022^{* *} \\
(0,0008)\end{array}$ & $\begin{array}{l}-0,0035^{* *} \\
(0,0008)\end{array}$ \\
\hline Arbeitslosenquote & & $\begin{array}{l}-0,026^{* * *} \\
(0,004)\end{array}$ & $\begin{array}{l}-0,020 * * * \\
(0,004)\end{array}$ & $\begin{array}{l}-0,013^{* * *} \\
(0,004)\end{array}$ & \\
\hline Frauenerwerbsquote & & $\begin{array}{l}-0,0083^{* * *} \\
(0,0026)\end{array}$ & $\begin{array}{l}-0,0082^{* * *} \\
(0,0025)\end{array}$ & & \\
\hline $\begin{array}{l}\text { Bevölkerungsdichte } \\
\text { logarithmiert }\end{array}$ & & & $\begin{array}{l}0,030 * * * \\
(0,009)\end{array}$ & $\begin{array}{l}0,032^{* * *} \\
(0,009)\end{array}$ & $\begin{array}{l}0,050^{* * *} \\
(0,007)\end{array}$ \\
\hline $\begin{array}{l}\text { Geschlechterproportion } \\
20-39\end{array}$ & & & $\begin{array}{l}-0,44^{*} \\
(0,20)\end{array}$ & $\begin{array}{l}-0,40^{*} \\
(0,20)\end{array}$ & \\
\hline Konstante & $\begin{array}{l}1,617^{* * *} \\
(0,024)\end{array}$ & $\begin{array}{l}2,617^{* * *} \\
(0,219)\end{array}$ & $\begin{array}{l}3,101 * * * \\
(0,289)\end{array}$ & $\begin{array}{l}2,388^{* * *} \\
(0,191)\end{array}$ & $\begin{array}{l}2,045^{* * *} \\
(0,054)\end{array}$ \\
\hline$\overline{\mathrm{R}^{2}}$ & $0,355 * * *$ & $0,498^{* * *}$ & $0,523 * * *$ & $0,507 * * *$ & $0,484^{* * *}$ \\
\hline Adj. $R^{2}$ & $0,349 * *$ & $0,489 * * *$ & $0,513^{* * *}$ & $0,498 * * *$ & $0,477^{* * *}$ \\
\hline $\mathrm{N}$ & 325 & 325 & 325 & 325 & 325 \\
\hline
\end{tabular}

Anmerkungen: Unter den Koeffizienten steht in Klammern der Standardfehler. *** $\mathrm{p}<0.001$; ** $\mathrm{p}<0.01 ;{ }^{*} \mathrm{p}<0,05$.

Quelle: Eigene Berechnung basierend auf Zensus 2011 und BBSR (2015).

Die Frauenerwerbsquote und die Katholikenquote erklären eine ähnliche Varianz, letztere ist nur dann signifikant, wenn erstere nicht inkludiert ist (Modelle W4-W5). Zwei für Gesamtdeutschland signifikante Variablen, die letztendlich aufgrund von hohen Interkorrelationen mit der Bevölkerungsdichte nicht berücksichtigt wurden, wurden zusätzlich getestet (Modelle nicht gezeigt): Der Migrationseffekt ist signifikant positiv, wenn der Migrantinnenanteil zusammen mit der Bevölkerungsdichte berücksichtigt wird. Der positive Effekt der Wohnungsgröße ist hochsignifikant, wenn diese Variable statt der Bevölkerungsdichte inkludiert wird. 
Tab. 6: OLS-Regressionen zur CTFR auf Kreisebene: Ostdeutschland

\begin{tabular}{|c|c|c|c|c|c|}
\hline & Modell 01 & Modell O2 & Modell O3 & Modell O4 & Modell O5 \\
\hline Anteil hoher & -0.0026 & & & & \\
\hline Bildungsabschluss & $(0.0020)$ & & & & \\
\hline $\begin{array}{l}\text { Anteil Migrations- } \\
\text { hintergrund }\end{array}$ & $\begin{array}{l}-0.0051 \\
(0.0038)\end{array}$ & & & & \\
\hline Anteil Katholiken & $\begin{array}{c}0.0028 \\
(0.0015)\end{array}$ & $\begin{array}{c}0.0026 \\
(0.0014)\end{array}$ & $\begin{array}{c}0.0025 \\
(0.0014)\end{array}$ & $\begin{array}{l}0.0029 * \\
(0.0014)\end{array}$ & $\begin{array}{c}0.0023 \\
(0.0014)\end{array}$ \\
\hline $\begin{array}{l}\text { Anteil evangelischer } \\
\text { Christen }\end{array}$ & $\begin{array}{l}0.0051 * * \\
(0.0016)\end{array}$ & $\begin{array}{c}0.0043^{*} \\
(0.0019)\end{array}$ & $\begin{array}{l}0.0052^{* *} \\
(0.0018)\end{array}$ & $\begin{array}{l}0.0054^{* *} \\
(0.0018)\end{array}$ & $\begin{array}{l}0.0046^{*} \\
(0.0019)\end{array}$ \\
\hline Arbeitslosenquote & & $\begin{array}{c}0.0078 \\
(0.0047)\end{array}$ & $\begin{array}{c}0.0041 \\
(0.0050)\end{array}$ & $\begin{array}{c}0.0054 \\
(0.0049)\end{array}$ & $\begin{array}{l}0.0098^{*} \\
(0.0047)\end{array}$ \\
\hline $\begin{array}{l}\text { Beschäftigte tertiärer } \\
\text { Sektor }\end{array}$ & & $\begin{array}{l}-0.0039^{* *} \\
(0.0012)\end{array}$ & & & \\
\hline BIP & & & $\begin{array}{l}-0.0096 * * \\
(0.0032)\end{array}$ & & \\
\hline $\begin{array}{l}\text { Bevölkerungsdichte } \\
\text { logarithmiert }\end{array}$ & & & & $\begin{array}{l}-0.036^{* *} \\
(0.012)\end{array}$ & \\
\hline $\begin{array}{l}\text { Anteil Fünf- und mehr } \\
\text { Raum-Wohnungen }\end{array}$ & & & & & $\begin{array}{l}0.0055^{* *} \\
(0.0017)\end{array}$ \\
\hline Konstante & $\begin{array}{l}1.471^{* * *} \\
(0.041)\end{array}$ & $\begin{array}{l}1.559 * * * \\
(0.139)\end{array}$ & $\begin{array}{l}1.517^{* * *} \\
(0.135)\end{array}$ & $\begin{array}{l}1.504^{* * *} \\
(0.130)\end{array}$ & $\begin{array}{l}1.082^{* * *} \\
(0.101)\end{array}$ \\
\hline $\mathrm{R}^{2}$ & $0.269 * * *$ & $0.318 * * *$ & $0.307 * * *$ & $0.310 * * *$ & $0.317^{* * *}$ \\
\hline Adj. $R^{2}$ & $0.229 * * *$ & $0.280 * * *$ & $0.268^{* * *}$ & $0.272 * * *$ & $0.279 * * *$ \\
\hline$N$ & 77 & 77 & 77 & 77 & 77 \\
\hline
\end{tabular}

Anmerkungen: Unter den Koeffizienten steht in Klammern der Standardfehler. *** $\mathrm{p}<0.001$; ** $\mathrm{p}<0.01$; * $\mathrm{p}<0,05$.

Quelle: Eigene Berechnung basierend auf Zensus 2011 und BBSR (2015).

Die Regressionsanalysen für Ostdeutschland ${ }^{12}$ in Tabelle 6 zeigen, dass der Anteil evangelischer Christen in allen Modellen signifikant mit einer höheren CTFR assoziiert ist. Daneben gibt es ein Cluster von vier Faktoren, die untereinander hoch interkorrelieren und sich jeweils einzeln als hochsignifikant $(p<0,01)$ erweisen (Modelle O2-O5): Beschäftigte im tertiären Sektor, BIP, Bevölkerungsdichte und der Anteil von Fünf- und mehr Raum-Wohnungen. In weniger urbanen Kreisen ist die Wirt-

12 Die Ergebnisse für Ostdeutschland sind aufgrund der niedrigen Fallzahlen mit Vorsicht zu interpretieren. 
schaftsstruktur traditioneller, das BIP geringer und mehr Wohnraum vorhanden. In diesen Kreisen ist auch eine Geschlechterproportion von wenigen jungen Frauen häufig. Welcher dieser Faktoren ausschlaggebend ist, lässt sich aufgrund der Interkorrelationen und der geringen Fallzahl bei Ostdeutschland nicht interpretieren.

\section{$5 \quad$ Zusammenfassung und Diskussion}

Dieser Beitrag hat das Ziel, die Bedeutung regionaler Rahmenbedingungen für die Fertilität zu analysieren und Daten zur regionalen Kohortenfertilität auf Kreisebene in Deutschland zu generieren. Zum einen wurden basierend auf den Mikrodaten der Haushaltsstichprobe des Zensus 2011 sowie der Geburtenstatistik erstmalig Schätzwerte für die CTFR für alle 402 deutschen Kreise berechnet. Zum anderen wurde mittels linearer Regressionsanalysen, basierend auf Zensus- und INKAR-Daten, Faktoren der regionalen Fertilitätsunterschiede analysiert. Dabei konnte gezeigt werden, dass regionale demografische Faktoren wie Bevölkerungsdichte und Geschlechterproportionen sowie regionale ökonomische Variablen wie Wirtschaftsstruktur, Wohnraum und Arbeitslosigkeit zum Verständnis kreisspezifischer Fertilitätsunterschiede beitragen können. Gleichzeitig zeigen die Befunde, dass auch Kompositionseffekte bezüglich Bildung, Religion und Migrantenanteil mit kreisspezifischen Fertilitätsunterschieden assoziiert sind.

Die Unterschiede der geschätzten kreisspezifischen CTFR für die Kohorten 196972 sind enorm: Die Spannbreite liegt zwischen 2,01 in Cloppenburg und 1,05 in Passau. Die niedrigste durchschnittliche Kinderzahl haben Frauen in Großstädten. In 44 Kreisen liegt sie bei über 1,7, vor allem im Allgäu, in der Odenwaldregion, in Südfranken und im westlichen Niedersachsen. Die kreisspezifische Verteilung der CTFR unterscheidet sich fundamental von der Verteilung der TFR für vergleichbare Jahre (BBSR 2015); der Korrelationskoeffizient beträgt lediglich 0,56. Der Vergleich beider Indikatoren auf Kreisebene verdeutlicht, dass der der TFR immanente Timing-Effekt insbesondere in Ostdeutschland die Fertilität unterschätzt. Auch die niedrige TFR in Studentenstädten ist ein statistisches Artefakt, da Studenten temporär den Nenner für bestimmte altersbedingt noch kinderlose Gruppen erhöhen. Während bspw. die Heidelberger TFR viele Jahre um die 1,0 oszillierte, liegt die CTFR bei 1,36.

Die Befunde der multivariaten Analysen haben eine breite internationale Relevanz, da verschiedenste Faktoren, die in der Literatur diskutiert werden, kombiniert Berücksichtigung finden. Für Deutschland lässt sich konstatieren, dass die CTFR in den Kreisen signifikant höher ist,

- die einen geringen Akademikerinnenanteil aufweisen,

- deren Katholikenquote hoch ist,

- deren Wirtschaftsstruktur traditioneller ist (geringer tertiärer Sektor),

- deren Arbeitslosenquote geringer ist,

- die stärker ländlich geprägt sind, 
- deren Immobilienmarkt relativ viele große Wohnungen für Familien anbietet und

- die einen relativen Männerüberschuss aufweisen.

Die Befunde für Gesamtdeutschland sind erwartungsgemäß denen für Westdeutschland ähnlich, da ein Großteil der Kreise (81\%) westdeutsch ist. Allerdings unterscheiden sich die Ergebnisse für West- und Ostdeutschland teilweise grundlegend. In Ostdeutschland ist die CTFR in den Kreisen signifikant höher,

- in denen viele evangelische Christen leben und

- die einem Cluster aus niedriger Bevölkerungsdichte, traditionellerer Wirtschaftsstruktur, niedrigem Sozialprodukt und vieler großer Wohnungen angehören.

Der robuste fertilitätsmindernde Einfluss einer hohen Bevölkerungsdichte bestätigt damit im Grundsatz die Urbanisierungsthese. Dieser bleibt auch bei Berücksichtigung anderer Faktoren signifikant, wenngleich sich die Effektstärke bei Inklusion der Bildungsvariablen reduziert. Die erheblichen Stadt-Land-Unterschiede liegen demnach sowohl darin begründet, dass in Städten mehr Akademikerinnen leben (die zu späteren Erstgeburten neigen), als auch daran, dass dort aufgrund der geringeren Naherholungsfläche und eines größeren Angebots an außerfamilialen Freizeitoptionen weniger Familien mit Kindern wohnen. Dazu trägt auch der migrationsspezifische Selektionseffekt bei, der besagt, dass karriereorientierte Frauen häufiger in Städte migrieren und umgekehrt.

Dabei haben die Analysen ein Cluster von mehreren Variablen im breiteren Kontext von Urbanisierung identifiziert. Dies ist theoretisch interessant, da es einen Ansatzpunkt darstellt, die Urbanisierungsthese weiter zu differenzieren. Denn in urbanisierten Kreisen treffen knapperer Wohnraum (Fiori et al. 2014; Kulu/Vikat 2007; Kulu 2013), eine moderne dienstleistungsorientierte Wirtschaftsstruktur sowie teilweise Frauenüberschüsse und modernere kulturelle Werte aufeinander. Diese Faktoren regionaler Opportunitäten erhöhen die Erklärungskraft, wenn man sie zusammen mit der Bevölkerungsdichte in Modellen inkludiert. Die Befunde konkretisieren Sharlins (1986) These für das frühe 21. Jahrhundert, wonach die Urbanisierungsthese auf geografischen, demografischen, ökonomischen und kulturellen Faktoren beruht. Dazu kommen bildungsspezifische Kompositionseffekte. ${ }^{13}$ In diesem Artikel wurde nur eine erste, tentative Annährung vorgenommen. Jedoch könnte zukünftige Forschung die Mechanismen im Urbanisierungscluster weiter ergründen.

Bemerkenswert ist die hohe Erklärungskraft der Religionsvariablen - zumal sie nicht nur die häufig untersuchte Katholikenquote betrifft, sondern auch die ostdeutschen Protestanten. Der Effekt bei den katholisch geprägten Kreisen ist auch im Kontext von Kinderbetreuung zu sehen, die in diesen Kreisen vergleichsweise ge-

13 Ohne den Migranteneffekt, wonach fertile und kulturell traditioneller orientierte Migrantengruppen überproportional in Großstädten wohnen, wären die Stadt-Land-Differenzen sogar noch deutlich größer. 
ring ausgebaut ist. Dieser Befund steht im Widerspruch zu ländervergleichenden Analysen (Castles 2003), bei denen die Katholikenquote negativ mit der Fertilität assoziiert ist. Eine Erklärung könnte sein, dass der Kinderbetreuungsausbau auf Länderebene hochgradig mit der Konfession zusammenhängt (Bujard 2011), während das geringe Betreuungsangebot zu Beginn dieses Jahrhunderts und der Ausbau seit dem Jahr 2005 Phänomene sind, die in katholischen und evangelischen Kreisen in Westdeutschland in ähnlicher Weise zutreffen. Dabei stellt sich eine grundsätzliche Frage: Misst der Anteil einer religiösen Konfession überhaupt einen Kompositionseffekt oder handelt es sich um einen Indikator zur kulturellen Prägung einer Region, die ein konservativeres Familienbild transportiert und Kinderreichtum eher als etwas Erstrebenswertes sieht (vgl. bereits Wolf 1912). Hier sind weitere Analysen notwendig.

Auch die Geschlechterproportionen stehen mit der CTFR in Zusammenhang. Diese sind insbesondere in den ostdeutschen Kreisen, bei denen auf 80 bis 90 Frauen 100 Männer kommen, positiv für die Fertilität der Frauen. Anscheinend führt die günstige Situation auf dem Partnermarkt dazu, dass weniger Frauen ohne Partner und damit verbunden ohne Kinder bleiben. Eine interessante Frage wäre, inwieweit die Männer in solchen kompetitiven Partnermarktkonstellationen eher bereit sind eine Familie zu gründen, um eine Partnerin "sicherer" an sich zu binden. Eine alternative Erklärung ist der migrationsspezifische Selektionseffekt.

Bei den Variablen zum Kinderbetreuungsangebot lassen sich keine einheitlichen Effekte finden. Dies bedeutet jedoch keinesfalls, dass diese Faktoren, die sich zwischen den Kreisen erheblich unterscheiden, keinen Einfluss haben. Vielmehr lassen sich die Effekte von Kinderbetreuungsangebot und Arbeitslosigkeit nur sinnvoll analysieren, wenn ihre Veränderungen im Zeitverlauf quantifiziert werden (u.a. Bauernschuster et al. 2013). Eine wichtige Implikation für die Familienpolitik ist jedoch der große Einfluss des Wohnungsmarktes auf die Fertilität. Da der Rückgang kinderreicher Familien für mehr als zwei Drittel (68 \%) des CTFR-Rückgangs zwischen den Frauenjahrgängen 1933 und 1968 in Deutschland verantwortlich ist (Bujard/ Sulak 2016), ist es plausibel, dass die Knappheit an Wohnungen mit fünf und mehr Räumen zur niedrigen Geburtenrate beiträgt.

Die hier vorgenommenen Makroanalysen bieten erstmals einen Überblick zu den Faktoren der regionalen Unterschiede der Kohortenfertilität. Sie können jedoch keine Aussagen darüber machen, wie hoch die Varianzerklärung der Kompositionseffekte im Vergleich zu den regionalen Effekten ist. Dafür sind Mehrebenenmodelle notwendig, die die hierarchischen Unterschiede von Mikro- und Makro-Variablen adäquat nutzen. In der Literatur zu Deutschland wird - stärker als in der internationalen - den Kompositionseffekten eine dominierende Rolle für die regionalen Fertilitätsunterschiede zugesprochen (Hank 2002; Hank/Huinink 2015). Dies steht zunächst in Widerspruch zu dem hier gezeigten Einfluss von regionalen Faktoren. Bisherige Mehrebenenanalysen wurden mit Individualdatensätzen wie pairfam, DJI-Familiensurvey oder SOEP und der Zuspielung kreisspezifischer Faktoren vorgenommen. Dabei sind die Fallzahlen relativ gering, wenn man bedenkt, dass pro Kreis durchschnittlich 20 bis 50 individuelle Fälle vorliegen. Daher bleibt es eine spannende Frage, zu welchem Ergebnis Mehrebenenanalysen auf Basis des Zensus 
kommen. Allerdings erkauft man sich durch die etwa 500-fache Vergrößerung der Fälle den Nachteil eines Forschungsdesigns im Querschnitt, ${ }^{14}$ das allerdings für die Abschätzung der Varianzanteile geeignet ist.

Dieser Beitrag hat zwei Implikationen für die weitere Forschung: Erstens lassen sich die hier generierten Schätzwerte zur Kohortenfertilität für zukünftige Forschungsvorhaben verwenden. Sie ermöglichen ein besseres Verständnis der kreisspezifischen Fertilitätsunterschiede als die periodischen TFR. Die Daten ermöglichen, bei Makroanalysen auf Kreisebene die Kohortenfertilität zu berücksichtigen. Sie ermöglichen aber auch die Identifizierung spezieller regionaler Konstellationen, insbesondere die identifizierten Hochfertilitätsregionen im östlichen Teil von Baden-Württemberg, Südfranken und im Nordwesten von Nordrhein-Westfalen. Um deren Ursachen zu erforschen, sind tiefenschärfere Fallanalysen einzelner Kreise vielversprechend. Zweifellos ergänzen regionale Fertilitätsanalysen den Forschungsstand zur Fertilität.

Zweitens werfen die signifikanten Befunde zum Einfluss von Religion, von Geschlechterrelationen und vom Cluster des Urbanisierungskontextes neue Fragen hinsichtlich Methodik und Theoriebildung auf, gerade wenn es darum geht, die Anteile von Kompositionseffekten und regionalem Kontext an der Gesamterklärung der CTFR abzuschätzen. Ein Problem ist dabei die fehlende Trennschärfe zwischen einigen Variablen, die sowohl individuell als auch strukturell wirken können. Ein Beispiel hierfür ist der Anteil von Katholiken, da die katholische Konfession ein individuelles Merkmal ist, der Faktor aber aggregiert die katholische Prägung und damit einen wichtigen kulturellen Faktor misst. Insgesamt liefert der Beitrag neue Erkenntnisse über den Zusammenhang zwischen regionalen Kontexten und Kohortenfertilität.

\section{Danksagung}

Wir danken Sebastian Klüsener, Stephan Kühntopf, Ralina Panova, Manfred Scharein und Felix zur Nieden sowie den beiden anonymen Gutachtern für wertvolle inhaltliche Hinweise. Ebenso danken wir Kai Dreschmitt für Literaturrecherche und dem Team des Zensus im Statistischen Bundesamt, insbesondere Sabrina Estatico, für datenspezifische Hinweise.

14 Dadurch lässt sich bspw. nicht klären, inwieweit der Urbanisierungseffekt durch Binnenmobilität zwischen Stadt und Land verursacht wird oder inwieweit durch das generative Verhalten von Frauen, die konstant in Großstädten leben (Boyle et al. 2007; Kulu/Boyle 2009). 


\section{Literatur}

Andorka, Rudolf 1978: Determinants of Fertility in Advanced Societies. London: Methuen \& Co.

Baizán, Pau 2009: Regional child care availability and fertility decisions in Spain. In: Demographic Research 21,27: 803-842 [doi: 10.4054/DemRes.2009.21.27].

Basten, Stuart; Huinink, Johannes; Klüsener, Sebastian 2011: Spatial Variation of Subnational Fertility Trends in Austria, Germany and Switzerland. In: Comparative Population Studies 36,2-3: 573-614 [doi: 10.4232/10.CPoS-2011-08en].

Bauernschuster, Stefan; Hener, Timo; Rainer, Helmut 2013: Does expanding public child care encourage fertility? County-level evidence from Germany. Ifo Working Paper 158.

BBSR (Bundesinstitut für Bau-, Stadt- und Raumforschung) (Hrsg.) 2015: Indikatoren und Karten zur Raum- und Stadtentwicklung. INKAR [http://www.inkar.de/, 01.06.2016].

Bechtold, Sabine 2016: The 2011 Census Model in Germany. In: Comparative Population Studies 4: D1-D9 [doi: 10.12765/CPoS-2016-07en].

Bongaarts, John; Feeney, Griffith 1998: On the Quantum and Tempo of Fertility. In: Population and Development Review 24,2: 271-291.

Boyle, Paul J.; Graham, Elspeth; Feng, Zhiqiang 2007: The significance of local clusters of fertility in Scotland. MPIDR Working paper WP 2007-036.

Bujard, Martin 2011: Geburtenrückgang und Familienpolitik. Baden-Baden: Nomos.

Bujard, Martin; Sulak, Harun 2016: Mehr Kinderlose oder weniger Kinderreiche? In: Kölner Zeitschrift für Soziologie und Sozialpsychologie 68,3: 487-514.

Castles, Francis G. 2003: The world turned upside down: Below replacement fertility, changing preferences and family-friendly public policy in 21 OECD countries. In: Journal of European Social Policy 13,3: 209-227 [doi: 10.1177/09589287030133001].

Coale, Ansley J.; Watkins, Susan C. 1986: The Decline of Fertility in Europe. Princeton: University Press.

Courgeau, Danie/ 1989: Family formation and urbanization. In: Population 44,1: 123-146.

Fiori, Francesca; Graham, Elspeth; Feng, Zhiqiang 2014: Geographical variations in fertility and transition to second and third birth in Britain. In: Advances in Life Course Research 21: 149-167 [doi: 10.1016/j.alcr.2013.11.004].

Fotheringham, A. Stewart; Wong, David W.S. 1991: The Modifiable Areal Unit Problem in Multivariate Statistical Analysis. In: Environment and Planning A 23: 1025-1044 [doi: 10.1068/a231025].

Fulda, Barbara 2015: Culture's Influence. Regionally Differing Social Milieus and Variations in Fertility Rates. MPIfG Discussion Paper 15/4.

Goldstein, Joshua R.; Klüsener, Sebastian 2014: Spatial analysis of the causes of fertility decline in Prussia. In: Population and Development Review 40,3: 497-525 [doi: 10.1111/j.1728-4457.2014.00695.x].

Goldstein, Joshua R.; Kreyenfeld, Michaela 2011: Has East Germany overtaken West Germany? Recent trends in order-specific fertility. In: Population and Development Review 37,3: 453-472 [doi: 10.1111/j.1728-4457.2011.00430.x].

Goldstein, Joshua R.; Kreyenfeld, Michaela 2013: Cohort_DATA_Germany_HFD. Unpublished data sheet.

Grünheid, Evelyn 2015: Regionale Aspekte des demografischen Wandels. Wiesbaden: Bundesinstitut für Bevölkerungsfoschung. 
Hank, Karsten 2001: Regional fertility differences in Western Germany: An Overview of the Literature and Recent Descriptive Findings. In: International Journal of Population Geography 7,4: 243-257.

Hank, Karsten 2002: Regional Social Contexts and Individual Fertility Decisions: A Multilevel Analysis of First and Second Births in Western Germany. In: European Journal of Population 18: 281-299 [doi: 10.1023/A:1019765026537].

Hank, Karsten 2003: The Differential Influence of Women's Residential District on the Risk of Entering First Marriage and Motherhood in Western Germany. In: Population and Environment 25: 3-21 [doi: 10.1023/A:1025546221461].

Hank, Karsten; Huinink, Johannes 2015: Regional Contexts and Family Formation. In: Hank, Karsten; Kreyenfeld, Michaela (Hrsg.): Social demography. Kölner Zeitschrift für Soziologie und Sozialpsychologie, Sonderheft 55. Wiesbaden: Springer VS: 41-58.

Hank, Karsten; Kreyenfeld, Michaela; Spieß, C. Katharina 2004: Kinderbetreuung und Fertilität in Deutschland. In: Zeitschrift für Soziologie 33: 228-244.

Huinink, Johannes; Wagner, Michael 1989: Regionale Lebensbedingungen, Migration und Familienbildung. In: Kölner Zeitschrift für Soziologie und Sozialpsychologie 41: 669-689.

Kemper, Franz-Josef 1991: Recent developments in household and family structure and their impact on regional fertility differences. In: Bähr, Jürgen; Gans, Paul (Hrsg.): The Geographical Approach to fertility. Kiel: 219-228.

Klüsener, Sebastian 2009: An Alternative Framework for Studying the Effects of Family Policies on Fertility in the Absence of Individual-Level Data. MPIDR WP 2009-027.

Klüsener, Sebastian; Goldstein, Joshua R. 2014: A Long-Standing Demographic EastWest Divide in Germany. In: Population, Space, and Place 22,1: 5-22 [doi: 10.1002/ psp.1870].

Knodel, John; van de Walle, Etienne 1986: Lessons from the Past: Policy Implications of Historical Fertility Studies. In: Coale, Ansley J.; Watkins, Susan C. (Hrsg.): The Decline of Fertility in Europe. Princeton: University Press: 390-419.

Kravdal, Øystein 2002: The impact of individual and aggregate unemployment on fertility in Norway. In: Demographic Research 6,10: 263-294 [doi: 10.4054/DemRes.2002.6.10].

Kreuzmair, Ingrid; Reisch, Marco 2013: ZENSUS 2011. Ablauf der Haushaltegenerierung [https://www.zensus2011.de/SharedDocs/Downloads/DE/Publikationen/Aufsaetze Archiv/2013_04_BW_Ablauf_der_Haushaltegenerierung.html, 01.06.2016].

Kühntopf, Stephan 2015: Kreisumschätzer. Unveröffentlichte Tabelle. Wiesbaden: BiB.

Kulu, Hill 2013: Why Do Fertility Levels Vary between Urban and Rural Areas? In: Regional Studies 47,6: 1-18.

Kulu, Hill; Milewski, Nadja 2007: Family change and migration in the life course: An introduction. In: Demographic Research 17,19: 567-590 [doi: 10.4054/DemRes.2007.17.19].

Kulu, Hill; Vikat, Andres; Andersson, Gunnar 2007: Settlement size and fertility in the Nordic countries. In: Population Studies 61,3: 265-285 [doi: 10.1080/00324720701571749].

Kulu, Hill; Vikat, Andres 2007: Fertility differences by housing type: The effect of housing conditions or of selective moves? In: Demographic Research 17,26: 775-802 [doi: 10.4054/DemRes.2007.17.26].

Kulu, Hill; Boyle, Paul J. 2009: High Fertility in City Suburbs: Compositional or Contextual Effects? In: European Journal of Population 25,2: 157-174 [doi: 10.1007/s10680008-9163-9]. 
Kulu, Hill; Washbrook, Elizabeth 2014: Residential context, migration and fertility in a modern urban society. In: Advances in Life Course Research 21: 168-182 [doi: 10.1016/j.alcr.2014.01.001].

Lesthaeghe, Ron 1995: The Second Demographic Transition in Western Countries: An Interpretation. In: Mason, Karen O.; Jensen, An-Magritt (Hrsg.): Gender and Family Change in Industrialized Countries. Oxford: Clarendon Press: 17-62.

Luci-Greulich, Angela; Thévenon, Olivier 2013: The impact of family policies on fertility trends in developed countries. In: European Journal of Population 29,4: 387-416 [doi: 10.1007/s10680-013-9295-4].

Lutz, Wolfgang; Testa, Maria R.; Penn, Dustin 2006: Population density is a key factor in declining human fertility. In: Population and Environment 28,2: 69-81 [doi: 10.1007/ s11111-007-0037-6].

Luy, Marc 2010: Tempo Effects and their Relevance in Demographic Analysis. In: Comparative Population Studies 35,3: 415-446 [doi: 10.4232/10.CPoS-2010-11en].

Michielin, Francesca 2002: Lowest low fertility in an urban context. When migration plays a key role. MPIDR Working paper WP 2002-050.

Mills, Melinda; Blossfeld, Hans-Peter 2005: Globalization, Uncertainty and the Early Life Course: A Theoretical Framework. In: Blossfeld, Hans-Peter et al. (Hrsg.): Globalization, Uncertainty and Youth in Society. London: Routledge: 1-14.

Openshaw, Stan 1984: The Modifiable Areal Unit Problem. Norwich: Geo Books.

Pötzsch, Olga 2010: Cohort fertility: A Comparison of the Results of the Official Birth Statistics and of the Microcensus Survey 2008. In: Comparative Population Studies 35,1: 185-204 [doi: 10.4232/10.CPoS-2010-05en].

Rindfuss, Ronald R. et al. 2007: Child Care Availability and First-Birth Timing in Norway. In: Demography 44,2: 345-372 [doi: 10.1353/dem.2007.0017].

Rokkan, Stein 1970: Citizens Elections Parties. Approaches to the Comparative Study of the Processes of Development. Oslo: Universitetsforlaget.

Schneider, Norbert F.; Naderi, Robert; Ruppenthal, Silvia 2012: Familie in Deutschland nach dem gesellschaftlichen Umbruch. In: Huinink, Johannes; Kreyenfeld, Michaela; Trappe, Heike (Hrsg.): Familie und Partnerschaft in Ost- und Westdeutschland: 29-53.

Schoen, Robert 2004: Timing effects and the interpretation of period fertility. In: Demography 41,4: 801-819 [doi: 10.1353/dem.2004.0036].

Sharlin, Allan 1986: Urban-Rural Differences in Fertility in Europe during the Demographic Transition. In: Coale, Ansley J.; Watkins, Susan C. (Hrsg.): The Decline of Fertility in Europe. Princeton: University Press: 234-260.

Sobotka, Tomáš; Feray, Adigüze/ 2002: Religiosity and Spatial Demographic Differences in the Netherlands. SOM Research Report. Groningen: University of Groningen.

Statistisches Bundesamt 2010: Haushaltebefragung beim Zensus 2011. Wiesbaden.

Statistisches Bundesamt 2012: Bevölkerung und Erwerbstätigkeit. Fachserie 1 Reihe 1.1. Wiesbaden.

Statistisches Bundesamt 2014: Altersspezifische Geburtenziffern der Geburtsjahrgänge 1930 bis 1997. Wiesbaden.

Statistisches Bundesamt 2015: Die Haushaltegenerierung - mehr als nur die Bildung von Haushalten [https://www.zensus2011.de/DE/Zensus2011/Methode/Methode_ Haushaltegenerierung_node.html, 01.06.2016]. 
Trovato, Frank; Grindstaff, Carl F. 1980: Decomposing the Urban-Rural Fertility Differential: Canada, 1971. In: Rural Sociology 45: 448-468.

Wolf, Julius 1912: Der Geburtenrückgang. Die Rationalisierung des Sexuallebens in unserer Zeit. Jena: Gustav Fischer.

Übersetzung des Originaltextes durch das Bundesinstitut für Bevölkerungsforschung, nur zur Information. Der begutachtete und von den Autoren autorisierte englische Originalbeitrag ist unter dem Titel Impact of Regional Factors on Cohort Fertility: New Estimations at the District Level in Germany", DOI 10.12765/CPoS-2017-07en bzw. URN urn:nbn:de:bib-cpos-2017-07en7, auf http:// www.comparativepopulationstudies.de verfügbar.

Dr. Martin Bujard $(\bowtie)$. Bundesinstitut für Bevölkerungsforschung. Wiesbaden, Deutschland.E-Mail: martin.bujard@bib.bund.de

URL: http://www.bib-demografie.de/bujard

Melanie Scheller. Statistisches Bundesamt. Wiesbaden, Deutschland.

E-Mail: melanie.scheller@destatis.de

URL: https://www.destatis.de/DE/Startseite.html 


\section{Anhang}

Abb. A1: Nummerierter Schlüssel der 402 deutschen Kreise

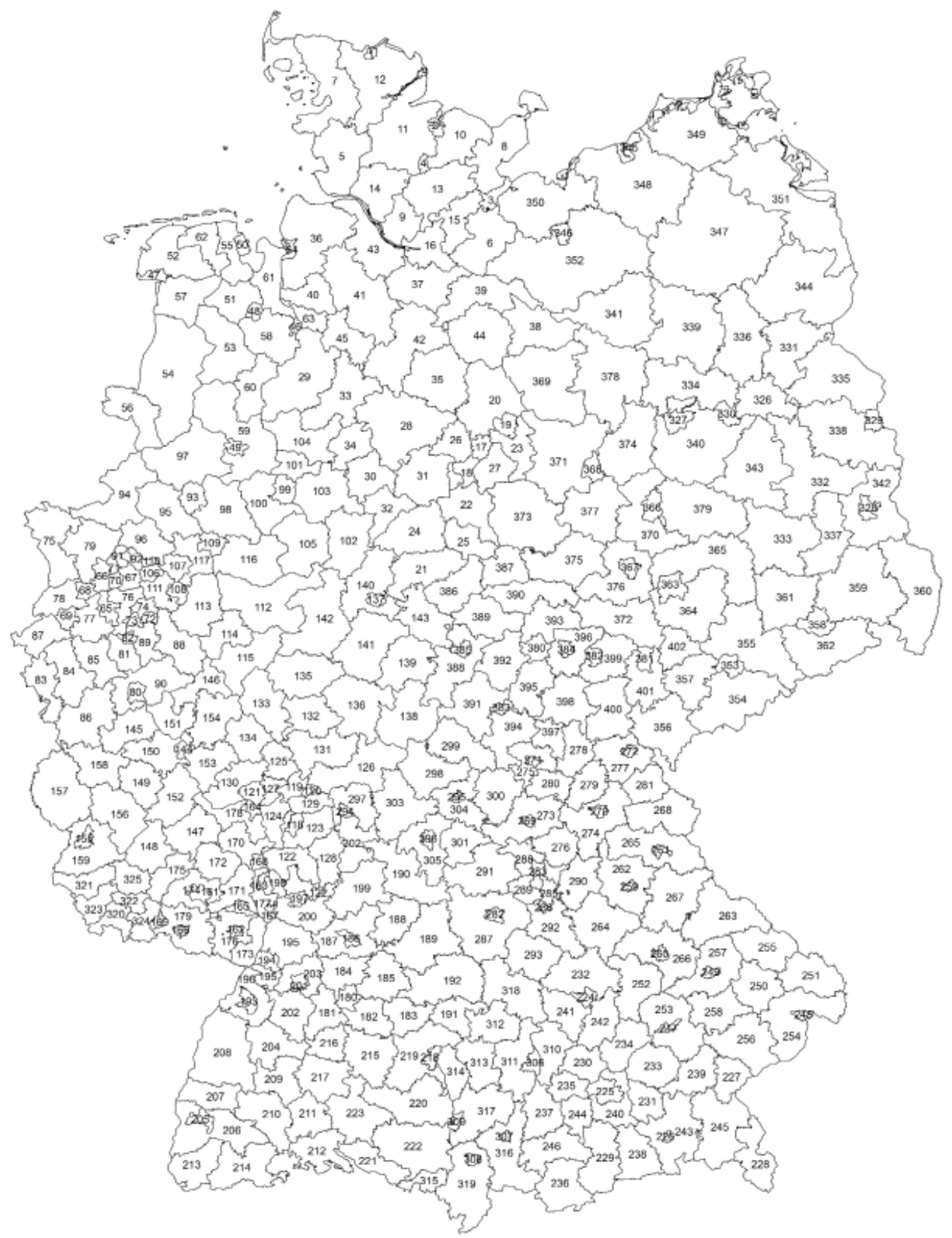

Quelle: Eigene Berechnungen. Karte: (c) GeoBasis-DE/BKG (2015). 
Tab. A1: Name, nummerierter Schlüssel und geschätzte CTFR für die Geburtskohorten 1969-72 der 402 deutschen Kreise

\begin{tabular}{|c|c|c|c|c|c|c|c|c|}
\hline Schlüssel & Kreis & CTFR & Schlüssel & Kreis & CTFR & Schlüssel & Kreis & CTFR \\
\hline 1 & Flensburg, Stadt & 1,39 & 51 & Ammerland & 1,63 & 101 & Herford & 1,48 \\
\hline 2 & Kiel, Landeshauptstadt & 1,11 & 52 & Aurich & 1,60 & 102 & Höxter & 1,66 \\
\hline 3 & Lübeck, Hansestadt & 1,30 & 53 & Cloppenburg & 2,01 & 103 & Lippe & 1,55 \\
\hline 4 & Neumünster, Stadt & 1,31 & 54 & Emsland & 1,82 & 104 & Minden-Lübbecke & 1,66 \\
\hline 5 & Dithmarschen & 1,50 & 55 & Friesland & 1,60 & 105 & Paderborn & 1,67 \\
\hline 6 & Herzogtum Lauenburg & 1,53 & 56 & Grafschaft Bentheim & 1,67 & 106 & Bochum, Stadt & 1,36 \\
\hline 7 & Nordfriesland & 1,53 & 57 & Leer & 1,67 & 107 & Dortmund, Stadt & 1,42 \\
\hline 8 & Ostholstein & 1,43 & 58 & Oldenburg & 1,47 & 108 & Hagen, Stadt & 1,59 \\
\hline 9 & Pinneberg & 1,42 & 59 & Osnabrück & 1,72 & 109 & Hamm, Stadt & 1,63 \\
\hline 10 & Plön & 1,48 & 60 & Vechta & 1,86 & 110 & Herne, Stadt & 1,58 \\
\hline 11 & Rendsburg-Eckernförde & 1,53 & 61 & Wesermarsch & 1,61 & 111 & Ennepe-Ruhr-Kreis & 1,39 \\
\hline 12 & Schleswig-Flensburg & 1,57 & 62 & Wittmund & 1,55 & 112 & Hochsauerlandkreis & 1,70 \\
\hline 13 & Segeberg & 1,43 & 63 & Bremen, Stadt & 1,28 & 113 & Märkischer Kreis & 1,45 \\
\hline 14 & Steinburg & 1,34 & 64 & Bremerhaven, Stadt & 1,43 & 114 & Olpe & 1,76 \\
\hline 15 & Stormarn & 1,43 & 65 & Düsseldorf, Stadt & 1,18 & 115 & Siegen-Wittgenstein & 1,61 \\
\hline 16 & Hamburg & 1,27 & 66 & Duisburg, Stadt & 1,56 & 116 & Soest & 1,61 \\
\hline 17 & Braunschweig, Stadt & 1,37 & 67 & Essen, Stadt & 1,29 & 117 & Unna & 1,57 \\
\hline 18 & Salzgitter, Stadt & 1,50 & 68 & Krefeld, Stadt & 1,57 & 118 & Darmstadt, Stadt & 1,44 \\
\hline 19 & Wolfsburg, Stadt & 1,53 & 69 & Mönchengladbach, Stadt & 1,49 & 119 & Frankfurt am Main, Stadt & 1,20 \\
\hline 20 & Gifhorn & 1,63 & 70 & Mülheim/Ruhr, Stadt & 1,37 & 120 & Offenbach am Main, Stadt & 1,41 \\
\hline 21 & Göttingen & 1,49 & 71 & Oberhausen, Stadt & 1,43 & 121 & Wiesbaden, Stadt & 1,31 \\
\hline 22 & Goslar & 1,47 & 72 & Remscheid, Stadt & 1,47 & 122 & Bergstraße & 1,42 \\
\hline 23 & Helmstedt & 1,51 & 73 & Solingen, Stadt & 1,52 & 123 & Darmstadt-Dieburg & 1,42 \\
\hline 24 & Northeim & 1,67 & 74 & Wuppertal, Stadt & 1,61 & 124 & Groß-Gerau & 1,48 \\
\hline 25 & Osterode am Harz & 1,55 & 75 & Kleve & 1,63 & 125 & Hochtaunuskreis & 1,49 \\
\hline 26 & Peine & 1,60 & 76 & Mettmann & 1,39 & 126 & Main-Kinzig-Kreis & 1,48 \\
\hline 27 & Wolfenbüttel & 1,47 & 77 & Rhein-Kreis Neuss & 1,49 & 127 & Main-Taunus-Kreis & 1,47 \\
\hline 28 & Region Hannover & 1,40 & 78 & Viersen & 1,51 & 128 & Odenwaldkreis & 1,60 \\
\hline 29 & Diepholz & 1,59 & 79 & Wesel & 1,54 & 129 & Offenbach & 1,38 \\
\hline 30 & Hameln-Pyrmont & 1,45 & 80 & Bonn, Stadt & 1,34 & 130 & Rheingau-Taunus-Kreis & 1,42 \\
\hline 31 & Hildesheim & 1,51 & 81 & Köln, Stadt & 1,19 & 131 & Wetteraukreis & 1,46 \\
\hline 32 & Holzminden & 1,56 & 82 & Leverkusen, Stadt & 1,36 & 132 & Gießen & 1,45 \\
\hline 33 & Nienburg (Weser) & 1,53 & 83 & Aachen & 1,46 & 133 & Lahn-Dill-Kreis & 1,61 \\
\hline 34 & Schaumburg & 1,61 & 84 & Düren & 1,56 & 134 & Limburg-Weilburg & 1,61 \\
\hline 35 & Celle & 1,65 & 85 & Rhein-Erft-Kreis & 1,56 & 135 & Marburg-Biedenkopf & 1,55 \\
\hline 36 & Cuxhaven & 1,51 & 86 & Euskirchen & 1,50 & 136 & Vogelsbergkreis & 1,65 \\
\hline 37 & Harburg & 1,37 & 87 & Heinsberg & 1,57 & 137 & Kassel, Stadt & 1,48 \\
\hline 38 & Lüchow-Dannenberg & 1,24 & 88 & Oberbergischer Kreis & 1,65 & 138 & Fulda & 1,64 \\
\hline 39 & Lüneburg & 1,48 & 89 & Rheinisch-Bergisch. Kreis & 1,55 & 139 & Hersfeld-Rotenburg & 1,51 \\
\hline 40 & Osterholz & 1,46 & 90 & Rhein-Sieg-Kreis & 1,55 & 140 & Kassel & 1,43 \\
\hline 41 & Rotenburg (Wümme) & 1,69 & 91 & Bottrop, Stadt & 1,37 & 141 & Schwalm-Eder-Kreis & 1,53 \\
\hline 42 & Soltau-Fallingbostel & 1,53 & 92 & Gelsenkirchen, Stadt & 1,59 & 142 & Waldeck-Frankenberg & 1,46 \\
\hline 43 & Stade & 1,62 & 93 & Münster, Stadt & 1,44 & 143 & Werra-Meißner-Kreis & 1,64 \\
\hline 44 & Uelzen & 1,41 & 94 & Borken & 1,78 & 144 & Koblenz, Stadt & 1,35 \\
\hline 45 & Verden & 1,54 & 95 & Coesfeld & 1,65 & 145 & Ahrweiler & 1,54 \\
\hline 46 & Delmenhorst, Stadt & 1,64 & 96 & Recklinghausen & 1,46 & 146 & Altenkirchen (Westerwald) & 1,57 \\
\hline 47 & Emden, Stadt & 1,68 & 97 & Steinfurt & 1,76 & 147 & Bad Kreuznach & 1,41 \\
\hline 48 & Oldenburg, Stadt & 1,37 & 98 & Warendorf & 1,65 & 148 & Birkenfeld & 1,44 \\
\hline 49 & Osnabrück, Stadt & 1,21 & 99 & Bielefeld, Stadt & 1,40 & 149 & Cochem-Zell & 1,60 \\
\hline 50 & Wilhelmshaven, Stadt & 1,33 & 100 & Gütersloh & 1,62 & 150 & Mayen-Koblenz & 1,52 \\
\hline
\end{tabular}


Tab. A1: Fortsetzung

\begin{tabular}{|c|c|c|c|c|c|c|c|c|}
\hline Schlüssel & Kreis & CTFR & Schlüssel & Kreis & CTFR & Schlüssel & Kreis & CTFR \\
\hline 151 & Neuwied & 1,57 & 201 & Pforzheim, Stadt & 1,75 & 251 & Freyung-Grafenau & 1,61 \\
\hline 152 & Rhein-Hunsrück-Kreis & 1,59 & 202 & Calw & 1,71 & 252 & Kelheim & 1,71 \\
\hline 153 & Rhein-Lahn-Kreis & 1,48 & 203 & Enzkreis & 1,62 & 253 & Landshut & 1,64 \\
\hline 154 & Westerwaldkreis & 1,65 & 204 & Freudenstadt & 1,86 & 254 & Passau & 1,65 \\
\hline 155 & Trier, Stadt & 1,29 & 205 & Freiburg im Breisgau, Stadt & 1,32 & 255 & Regen & 1,51 \\
\hline 156 & Bernkastel-Wittlich & 1,66 & 206 & Breisgau-Hochschwarzwald & 1,63 & 256 & Rottal-Inn & 1,84 \\
\hline 157 & Bitburg-Prüm & 1,65 & 207 & Emmendingen & 1,62 & 257 & Straubing-Bogen & 1,84 \\
\hline 158 & Daun & 1,83 & 208 & Ortenaukreis & 1,62 & 258 & Dingolfing-Landau & 1,66 \\
\hline 159 & Trier-Saarburg & 1,56 & 209 & Rottweil & 1,70 & 259 & Amberg, Stadt & 1,31 \\
\hline 160 & Frankenthal (Pfalz), Stadt & 1,44 & 210 & Schwarzwald-Baar-Kreis & 1,63 & 260 & Regensburg, Stadt & 1,26 \\
\hline 161 & Kaiserslautern, Stadt & 1,26 & 211 & Tuttlingen & 1,77 & 261 & Weiden i.d.OPf., Stadt & 1,16 \\
\hline 162 & Landau in der Pfalz, Stadt & 1,46 & 212 & Konstanz & 1,47 & 262 & Amberg-Sulzbach & 1,61 \\
\hline 163 & Ludwigshafen am Rhein & 1,41 & 213 & Lörrach & 1,60 & 263 & Cham & 1,64 \\
\hline 164 & Mainz, Stadt & 1,42 & 214 & Waldshut & 1,56 & 264 & Neumarkt i.d.OPf. & 1,74 \\
\hline 165 & Neustadt Weinstraße, Stadt & 1,54 & 215 & Reutlingen & 1,60 & 265 & Neustadt a.d.Waldnaab & 1,73 \\
\hline 166 & Pirmasens, Stadt & 1,27 & 216 & Tübingen & 1,52 & 266 & Regensburg & 1,54 \\
\hline 167 & Speyer, Stadt & 1,60 & 217 & Zollernalbkreis & 1,67 & 267 & Schwandorf & 1,52 \\
\hline 168 & Worms, Stadt & 1,35 & 218 & Ulm, Universitätsstadt & 1,42 & 268 & Tirschenreuth & 1,78 \\
\hline 169 & Zweibrücken, Stadt & 1,40 & 219 & Alb-Donau-Kreis & 1,76 & 269 & Bamberg, Stadt & 1,21 \\
\hline 170 & Alzey-Worms & 1,58 & 220 & Biberach & 1,81 & 270 & Bayreuth, Stadt & 1,44 \\
\hline 171 & Bad Dürkheim & 1,54 & 221 & Bodenseekreis & 1,60 & 271 & Coburg, Stadt & 1,51 \\
\hline 172 & Donnersbergkreis & 1,56 & 222 & Ravensburg & 1,78 & 272 & Hof, Stadt & 1,38 \\
\hline 173 & Germersheim & 1,53 & 223 & Sigmaringen & 1,66 & 273 & Bamberg & 1,61 \\
\hline 174 & Kaiserslautern & 1,55 & 224 & Ingolstadt, Stadt & 1,41 & 274 & Bayreuth & 1,59 \\
\hline 175 & Kusel & 1,44 & 225 & München, Landeshauptstadt & 1,18 & 275 & Coburg & 1,63 \\
\hline 176 & Südliche Weinstraße & 1,51 & 226 & Rosenheim, Stadt & 1,39 & 276 & Forchheim & 1,68 \\
\hline 177 & Rhein-Pfalz-Kreis & 1,37 & 227 & Altötting & 1,63 & 277 & Hof & 1,45 \\
\hline 178 & Mainz-Bingen & 1,39 & 228 & Berchtesgadener Land & 1,51 & 278 & Kronach & 1,48 \\
\hline 179 & Südwestpfalz & 1,52 & 229 & Bad Tölz-Wolfratshausen & 1,64 & 279 & Kulmbach & 1,47 \\
\hline 180 & Stuttgart, Landeshauptstadt & 1,29 & 230 & Dachau & 1,53 & 280 & Lichtenfels & 1,78 \\
\hline 181 & Böblingen & 1,52 & 231 & Ebersberg & 1,61 & 281 & Wunsiedel i.Fichtelgebirge & 1,23 \\
\hline 182 & Esslingen & 1,49 & 232 & Eichstätt & 1,86 & 282 & Ansbach, Stadt & 1,43 \\
\hline 183 & Göppingen & 1,61 & 233 & Erding & 1,59 & 283 & Erlangen, Stadt & 1,32 \\
\hline 184 & Ludwigsburg & 1,55 & 234 & Freising & 1,55 & 284 & Fürth, Stadt & 1,31 \\
\hline 185 & Rems-Murr-Kreis & 1,55 & 235 & Fürstenfeldbruck & 1,44 & 285 & Nürnberg, Stadt & 1,26 \\
\hline 186 & Heilbronn, Stadt & 1,49 & 236 & Garmisch-Partenkirchen & 1,53 & 286 & Schwabach, Stadt & 1,34 \\
\hline 187 & Heilbronn & 1,70 & 237 & Landsberg a.Lech & 1,81 & 287 & Ansbach & 1,63 \\
\hline 188 & Hohenlohekreis & 1,78 & 238 & Miesbach & 1,57 & 288 & Erlangen-Höchstadt & 1,49 \\
\hline 189 & Schwäbisch Hall & 1,76 & 239 & Mühldorf a.Inn & 1,87 & 289 & Fürth & 1,40 \\
\hline 190 & Main-Tauber-Kreis & 1,75 & 240 & München & 1,51 & 290 & Nürnberger Land & 1,44 \\
\hline 191 & Heidenheim & 1,78 & 241 & Neuburg-Schrobenhausen & 1,60 & 291 & Neustadt a.d.Aisch-Bad W. & 1,57 \\
\hline 192 & Ostalbkreis & 1,76 & 242 & Pfaffenhofen a.d.IIm & 1,62 & 292 & Roth & 1,66 \\
\hline 193 & Baden-Baden, Stadt & 1,22 & 243 & Rosenheim & 1,69 & 293 & Weißenburg-Gunzenhausen & 1,73 \\
\hline 194 & Karlsruhe, Stadt & 1,26 & 244 & Starnberg & 1,56 & 294 & Aschaffenburg, Stadt & 1,50 \\
\hline 195 & Karlsruhe & 1,52 & 245 & Traunstein & 1,67 & 295 & Schweinfurt, Stadt & 1,77 \\
\hline 196 & Rastatt & 1,53 & 246 & Weilheim-Schongau & 1,71 & 296 & Würzburg, Stadt & 1,16 \\
\hline 197 & Heidelberg, Stadt & 1,36 & 247 & Landshut, Stadt & 1,27 & 297 & Aschaffenburg & 1,64 \\
\hline 198 & Mannheim, Stadt & 1,34 & 248 & Passau, Stadt & 1,05 & 298 & Bad Kissingen & 1,63 \\
\hline 199 & Neckar-Odenwald-Kreis & 1,84 & 249 & Straubing, Stadt & 1,60 & 299 & Rhön-Grabfeld & 1,64 \\
\hline 200 & Rhein-Neckar-Kreis & 1,49 & 250 & Deggendorf & 1,47 & 300 & Haßberge & 1,64 \\
\hline
\end{tabular}


Tab. A1: Fortsetzung

\begin{tabular}{|c|c|c|c|c|c|c|c|c|}
\hline Schlüssel & Kreis & CTFR & Schlüssel & Kreis & CTFR & Schlüssel & Kreis & CTFR \\
\hline 301 & Kitzingen & 1,77 & 351 & Vorpommern-Greifswald & 1,45 & 401 & Greiz & 1,67 \\
\hline 302 & Miltenberg & 1,60 & 352 & Ludwigslust-Parchim & 1,51 & 402 & Altenburger Land & 1,46 \\
\hline 303 & Main-Spessart & 1,57 & 353 & Chemnitz & 1,65 & & & \\
\hline 304 & Schweinfurt & 1,66 & 354 & Erzgebirgskreis & 1,71 & & & \\
\hline 305 & Würzburg & 1,51 & 355 & Mittelsachsen & 1,62 & & & \\
\hline 306 & Augsburg, Stadt & 1,28 & 356 & Vogtlandkreis & 1,56 & & & \\
\hline 307 & Kaufbeuren, Stadt & 1,54 & 357 & Zwickau & 1,56 & & & \\
\hline 308 & Kempten (Allgäu), Stadt & 1,45 & 358 & Dresden & 1,52 & & & \\
\hline 309 & Memmingen, Stadt & 1,50 & 359 & Bautzen & 1,61 & & & \\
\hline 310 & Aichach-Friedberg & 1,59 & 360 & Görlitz & 1,73 & & & \\
\hline 311 & Augsburg & 1,67 & 361 & Meißen & 1,66 & & & \\
\hline 312 & Dillingen a.d.Donau & 1,62 & 362 & Sächsische Schweiz-Oster. & 1,65 & & & \\
\hline 313 & Günzburg & 1,88 & 363 & Leipzig & 1,34 & & & \\
\hline 314 & Neu-Ulm & 1,74 & 364 & Leipzig & 1,51 & & & \\
\hline 315 & Lindau (Bodensee) & 1,50 & 365 & Nordsachsen & 1,62 & & & \\
\hline 316 & Ostallgäu & 1,76 & 366 & Dessau-Roßlau & 1,37 & & & \\
\hline 317 & Unterallgäu & 1,80 & 367 & Halle (Saale) & 1,40 & & & \\
\hline 318 & Donau-Ries & 1,65 & 368 & Magdeburg & 1,45 & & & \\
\hline 319 & Oberallgäu & 1,57 & 369 & Altmarkkreis Salzwedel & 1,62 & & & \\
\hline 320 & Stadtverband Saarbrücken & 1,34 & 370 & Anhalt-Bitterfeld & 1,47 & & & \\
\hline 321 & Merzig-Wadern & 1,57 & 371 & Börde & 1,53 & & & \\
\hline 322 & Neunkirchen & 1,46 & 372 & Burgenlandkreis & 1,51 & & & \\
\hline 323 & Saarlouis & 1,38 & 373 & Harz & 1,47 & & & \\
\hline 324 & Saarpfalz-Kreis & 1,39 & 374 & Jerichower Land & 1,38 & & & \\
\hline 325 & St. Wendel & 1,39 & 375 & Mansfeld-Südharz & 1,55 & & & \\
\hline 326 & Berlin & 1,37 & 376 & Saalekreis & 1,48 & & & \\
\hline 327 & Brandenburg an der Havel & 1,24 & 377 & Salzlandkreis & 1,46 & & & \\
\hline 328 & Cottbus, Stadt & 1,44 & 378 & Stendal & 1,58 & & & \\
\hline 329 & Frankfurt (Oder), Stadt & 1,34 & 379 & Wittenberg & 1,52 & & & \\
\hline 330 & Potsdam, Stadt & 1,35 & 380 & Erfurt, Stadt & 1,48 & & & \\
\hline 331 & Barnim & 1,47 & 381 & Gera, Stadt & 1,11 & & & \\
\hline 332 & Dahme-Spreewald & 1,50 & 382 & Jena, Stadt & 1,47 & & & \\
\hline 333 & Elbe-Elster & 1,68 & 383 & Suhl, Stadt & 1,39 & & & \\
\hline 334 & Havelland & 1,59 & 384 & Weimar, Stadt & 1,41 & & & \\
\hline 335 & Märkisch-Oderland & 1,49 & 385 & Eisenach, Stadt & 1,33 & & & \\
\hline 336 & Oberhavel & 1,48 & 386 & Eichsfeld & 1,66 & & & \\
\hline 337 & Oberspreewald-Lausitz & 1,64 & 387 & Nordhausen & 1,32 & & & \\
\hline 338 & Oder-Spree & 1,47 & 388 & Wartburgkreis & 1,68 & & & \\
\hline 339 & Ostprignitz-Ruppin & 1,53 & 389 & Unstrut-Hainich-Kreis & 1,60 & & & \\
\hline 340 & Potsdam-Mittelmark & 1,62 & 390 & Kyffhäuserkreis & 1,52 & & & \\
\hline 341 & Prignitz & 1,63 & 391 & Schmalkalden-Meiningen & 1,58 & & & \\
\hline 342 & Spree-Neiße & 1,52 & 392 & Gotha & 1,53 & & & \\
\hline 343 & Teltow-Fläming & 1,53 & 393 & Sömmerda & 1,63 & & & \\
\hline 344 & Uckermark & 1,42 & 394 & Hildburghausen & 1,54 & & & \\
\hline 345 & Rostock & 1,38 & 395 & IIm-Kreis & 1,44 & & & \\
\hline 346 & Schwerin & 1,41 & 396 & Weimarer Land & 1,44 & & & \\
\hline 347 & Mecklenburgische Seenplatte & 1,53 & 397 & Sonneberg & 1,33 & & & \\
\hline 348 & Landkreis Rostock & 1,58 & 398 & Saalfeld-Rudolstadt & 1,43 & & & \\
\hline 349 & Vorpommern-Rügen & 1,53 & 399 & Saale-Holzland-Kreis & 1,48 & & & \\
\hline 350 & Nordwestmecklenburg & 1,71 & 400 & Saale-Orla-Kreis & 1,49 & & & \\
\hline
\end{tabular}

Anmerkung: Ein Excelfile mit 5-stelliger Kreisnummer, Kreisname, Bundesland und dem CTFR-Schätzwert wird öffentlich bereitgestellt unter: http://www.comparativepopulationstudies.de/index.php/CPoS/article/view/278/251.

Quelle: Eigene Berechnungen basierend auf Zensus 2011. 


\section{Comparative Population Studies}

wWW.comparativepopulationstudies.de

ISSN: 1869-8980 (Print) - 1869-8999 (Internet)

\section{Published by}

Prof. Dr. Norbert F. Schneider

Federal Institute for Population Research D-65180 Wiesbaden / Germany

\section{(cc) BY-SA}

2017

\section{Managing Editor}

Frank Swiaczny

\section{Assistant Managing Editor}

Katrin Schiefer

\section{Copy Editor}

(Selected Articles in German)

Dr. Evelyn Grünheid

\section{Layout}

Beatriz Feiler-Fuchs

E-mail: cpos@bib.bund.de

\section{Scientific Advisory Board}

Paul Gans (Mannheim)

Karsten Hank (Cologne)

Johannes Huinink (Bremen)

Michaela Kreyenfeld (Rostock)

Marc Luy (Vienna)

Notburga Ott (Bochum)

Peter Preisendörfer (Mainz)

Nikola Sander (Groningen)

Zsolt Spéder (Budapest)

\section{Board of Reviewers}

Martin Abraham (Erlangen)

Laura Bernardi (Lausanne)

Hansjörg Bucher (Bonn)

Claudia Diehl (Konstanz)

Andreas Diekmann (Zurich)

Gabriele Doblhammer-Reiter (Rostock)

Jürgen Dorbritz (Wiesbaden)

Anette Eva Fasang (Berlin)

E.-Jürgen Flöthmann (Bielefeld)

Alexia Fürnkranz-Prskawetz (Vienna)

Beat Fux (Salzburg)

Joshua Goldstein (Berkeley)

Sonja Haug (Regensburg)

Hill Kulu (Liverpool)

Aart C. Liefbroer (The Hague)

Kurt Lüscher (Konstanz)

Emma Lundholm (Umeå)

Nadja Milewski (Rostock)

Dimiter Philipov (Vienna)

Roland Rau (Rostock)

Tomáš Sobotka (Vienna)

Jeroen Spijker (Barcelona)

Olivier Thévenon (Paris)

Helga de Valk (Brussels)

Heike Trappe (Rostock)

Michael Wagner (Cologne) 